\title{
COVID-19 in the Age of Artificial Intelligence: A Comprehensive Review
}

\author{
Jawad Rasheed $^{1}$ (1) $\cdot$ Akhtar Jamil $^{2} \cdot$ Alaa Ali Hameed $^{2} \cdot$ Fadi Al-Turjman $^{3} \cdot$ Ahmad Rasheed $^{4}$
}

Received: 29 September 2020 / Revised: 3 April 2021 / Accepted: 9 April 2021 / Published online: 22 April 2021

(C) International Association of Scientists in the Interdisciplinary Areas 2021

\begin{abstract}
The recent COVID-19 pandemic, which broke at the end of the year 2019 in Wuhan, China, has infected more than 98.52 million people by today (January 23, 2021) with over 2.11 million deaths across the globe. To combat the growing pandemic on urgent basis, there is need to design effective solutions using new techniques that could exploit recent technology, such as machine learning, deep learning, big data, artificial intelligence, Internet of Things, for identification and tracking of COVID-19 cases in near real time. These technologies have offered inexpensive and rapid solution for proper screening, analyzing, prediction and tracking of COVID-19 positive cases. In this paper, a detailed review of the role of AI as a decisive tool for prognosis, analyze, and tracking the COVID-19 cases is performed. We searched various databases including Google Scholar, IEEE Library, Scopus and Web of Science using a combination of different keywords consisting of COVID-19 and AI. We have identified various applications, where AI can help healthcare practitioners in the process of identification and monitoring of COVID-19 cases. A compact summary of the corona virus cases are first highlighted, followed by the application of AI. Finally, we conclude the paper by highlighting new research directions and discuss the research challenges. Even though scientists and researchers have gathered and exchanged sufficient knowledge over last couple of months, but this structured review also examined technological perspectives while encompassing the medical aspect to help the healthcare practitioners, policymakers, decision makers, policymakers, AI scientists and virologists to quell this infectious COVID-19 pandemic outbreak.
\end{abstract}

\section{Graphic abstract}

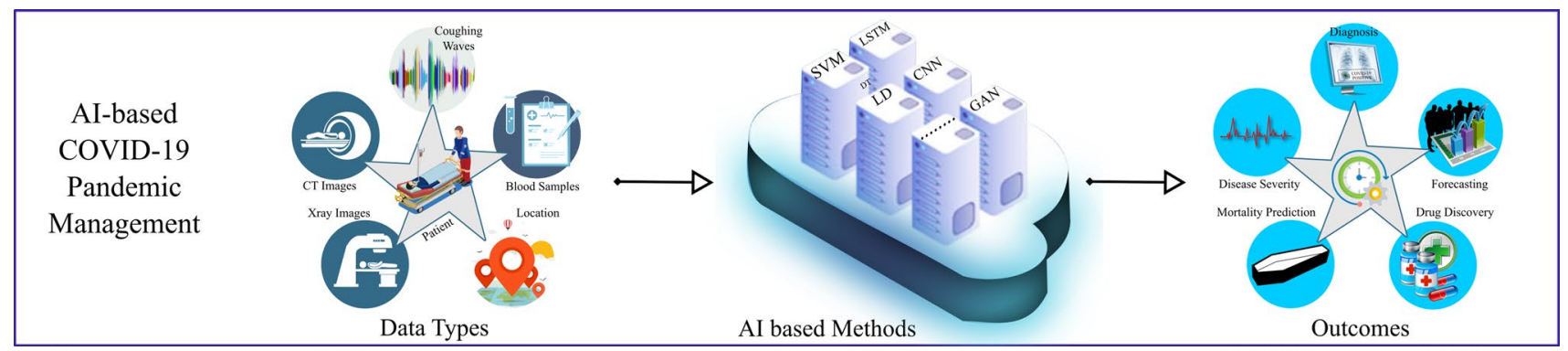

Keywords COVID-19 $\cdot$ Deep learning $\cdot$ Infectious diseases $\cdot$ Drug discovery $\cdot$ Disease prediction $\cdot$ Machine learning $\cdot$ SARS-CoV-2

\section{Abbreviations}

$\mathrm{AE}$

AI

ANFIS
Auto-encoders

Artificial intelligence

Adaptive network-based fuzzy inference system

Jawad Rasheed

jawadrasheed@aydin.edu.tr

Extended author information available on the last page of the article
ANN Artificial neural network

ARIMA

BI-AT-GRU

Autoregressive integrated moving average

Bidirectional and attentional gated recur-

rent unit neural network

BI-AT-LSTM Bidirectional and attentional long shortterm memory

CNN Convolutional neural networks

CPM-Nets Cross partial multi-view networks 


\begin{tabular}{|c|c|}
\hline CRP & C-reactive protein \\
\hline $\mathrm{CT}$ & Computed tomography \\
\hline $\mathrm{CV}$ & Computer vision \\
\hline CXR & Chest X-rays \\
\hline DL & Deep learning \\
\hline DT & Decision trees \\
\hline DTL & Deep transfer learning \\
\hline GANs & Generative adversarial networks \\
\hline GLCM & Gray level co-occurrence matrix \\
\hline GPU & Graphical processing unit \\
\hline GRU & Gated recurrent unit \\
\hline IAVP & Influenza-A viral pneumonia \\
\hline IoT & Internet of things \\
\hline KNN & K-nearest neighbor \\
\hline LD & Linear discriminant \\
\hline LNR & Linear regression \\
\hline LSTM & Long short-term memory \\
\hline LR & Logistic regression \\
\hline LUS & Lung ultrasound \\
\hline MAE & Mean absolute error \\
\hline MAPE & Mean absolute percent error \\
\hline MCDM & Multi-criteria decision making \\
\hline $\mathrm{MCV}$ & Mean corpuscular volume \\
\hline MERS-CoV & $\begin{array}{l}\text { Middle east respiratory syndrome } \\
\text { coronavirus }\end{array}$ \\
\hline ML & Machine learning \\
\hline MLP & Multi-layer perceptron \\
\hline NPV & Negative predicted value \\
\hline PCA & Principal component analysis \\
\hline PDR-NML & $\begin{array}{l}\text { Partial derivative regression and non- } \\
\text { linear machine learning }\end{array}$ \\
\hline PET & Positron emission tomography \\
\hline PHEIC & $\begin{array}{l}\text { Public-health emergency of international } \\
\text { concern }\end{array}$ \\
\hline PPDLR & $\begin{array}{l}\text { Progressive partial derivate linear } \\
\text { regression }\end{array}$ \\
\hline PPV & Positive predicted value \\
\hline $\mathrm{R}-\mathrm{CNN}$ & $\begin{array}{l}\text { Region-based convolutional neural } \\
\text { networks }\end{array}$ \\
\hline RDW & Red cell distribution width \\
\hline $\mathrm{RF}$ & Random forest \\
\hline RMSE & Root-mean-square error \\
\hline RNN & Recurrent neural network \\
\hline RSM & Respiratory stimulated model \\
\hline RT-PCR & $\begin{array}{l}\text { Reverse-transcription polymerase chain } \\
\text { reaction }\end{array}$ \\
\hline SARS & Severe acute respiratory syndrome \\
\hline SARS-CoV-2 & $\begin{array}{l}\text { Severe acute respiratory syndrome-coro- } \\
\text { navirus } 2\end{array}$ \\
\hline SEIR & Susceptible exposed infected recovered \\
\hline SMILE & $\begin{array}{l}\text { Simplified molecule input line entry } \\
\text { system }\end{array}$ \\
\hline SMOTE & Synthetic minority over-sample technique \\
\hline
\end{tabular}

$\begin{array}{ll}\text { SOM } & \text { Self-organizing maps } \\ \text { SVM } & \text { Support vector machine } \\ \text { TF-IDF } & \begin{array}{l}\text { Term frequency-inverse document } \\ \text { frequency }\end{array} \\ & \text { Variational auto-encoder } \\ \text { VAE } & \text { Visual geometry group } \\ \text { VGG-X } & \text { World Health Organization } \\ \text { WHO } & \text { Extreme gradient boosting } \\ \text { XGB } & \end{array}$

\section{Introduction}

COVID-19 is a highly contagious epidemic disease caused by novel coronavirus (SARS-CoV-2) which has been declared as pandemic by WHO. Researchers across the globe are working round the clock to find solutions and design strategies to control the pandemic and minimize its impact on human health and economy [1]. One of the large family of viruses is called Coronaviruses, which may affect and endanger humans' lives by causing acute ailment [2]. A virus is an infectious microorganism constitutes a specific genome wrapped in a protein layer with the ability to replicate inside living cells. These billions of tiny but powerful viruses, smaller than human cells, can cause viral infection when entered in living creature by hijacking the host cells and forcefully turning those to virus-making factory. This may lead to severe health problems, such as blindness due to smallpox virus or fatal inflammation of the brain or spinal cord by rabies virus $[3,4]$.

These viruses are either pandemic or epidemic that lasts over a definite duration of time [5]. A pandemic is an occurrence of huge morbidities and mortalities caused by the proliferation of infectious disease at vast geographical area. Contrarily, when a disease is spread in limited region over time [6]. Many epidemics and pandemics occurred over a period, but the mortality rate shows that pandemics had effects that are more devastating in the history of human life. Such as a decade ago, SARS epidemic virus infected around 8096 humans causing deaths of more than 770 humans. Beside such small epidemic, years ago, a famous pandemic known as smallpox affected millions of lives and eventually ended up with 500 million fatalities across the globe [7]. Few of the viruses that caused epidemic and pandemic over last 102 years are depicted in Fig. 1.

The novel Coronavirus is the recent pandemic, officially known as SARS-CoV-2 and member of broader family of infectious viruses, which can affect the respiratory system of humans [8]. In 2002, the first pathogen, SARS emerged in Guangdong (China) that caused mild infection in humans. Another pathogenic member of coronavirus family, known as MERS-CoV, discovered in 2012 around Middle East regions that caused panic due to high infection rate which affected 2494 humans with 858 deaths [9]. Last December 


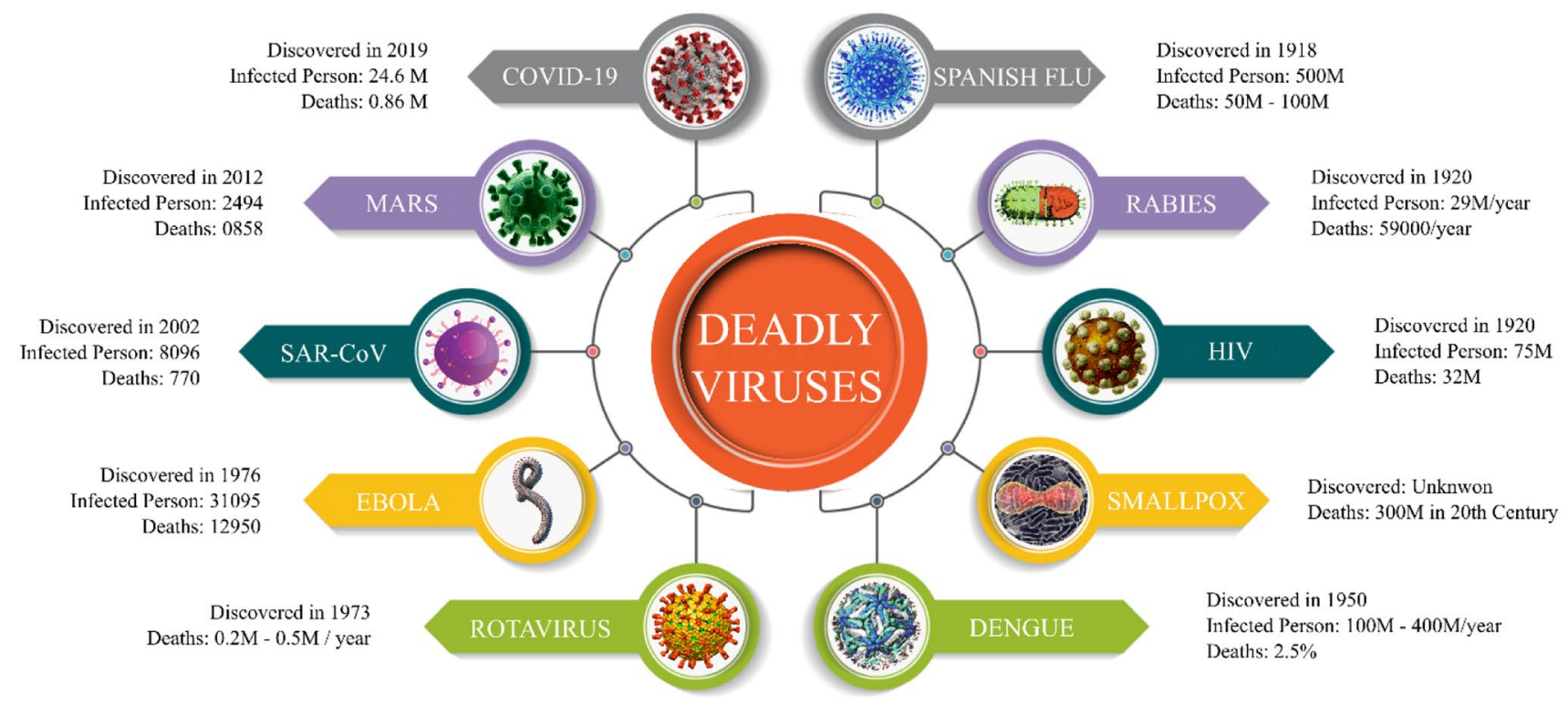

Fig. 1 Deadliest viruses over last 102 years (as of August 28, 2020)

(2019), the deadly COVID-19, the seventh strain of coronavirus, originated in Huanan Seafood Market, Wuhan state of Hubei province, China, that quickly gained global attention due to fast transmission among species and caused respiratory problems $[10,11]$. COVID-19 being an infectious disease has high transmission rate among humans as respiratory droplets of infected patient are inhaled by humans around [12]. Tiredness, cough, fever, and loss of smell are considered as most common symptoms, while headache, aches, rash on skin, diarrhea and sore throat are less common symptoms observed in patients affected by COVID- 19 . Besides these normal looking symptoms, it can cause severe respiratory problems that can damage several human organs, which eventually causes death $[13,14]$.

On January 30, 2020, due to high horizontal transmission rate among humans of 18 different countries, WHO announced it a PHEIC [15]. Keeping in view the sharp incline of COVID-19 cases around the world in succeeding 2 months, health organizations stated it as global pandemic due to its hazardous effect on human life [16]. Since the first outbreak of highly pathogenic in China, the pandemic has affected 213 countries and territories around the world according to figures compiled by Worldometer [17]. As reported by John Hopkins University this particular virus has infected more than $98,529,820$ humans around the globe, while the tally of confirmed deaths is over 2,116,101 [18], thus becoming the greatest pandemic of all time. As per John Hopkins data, a total number of infections and deaths in top 10 disease burden countries are shown in Fig. 2.

The early identification of COVID-19 cases is momentous as it not only helps start treatment of the cases immediately, but also facilitates containing the virus by isolating the patient from other humans. Presently, RT-PCR is considered as the established procedure to identify the positive cases of COVID-19. To further speed up the identification operations, there is still room for the advancement of better auxiliary alternative diagnostic tools to enhance the identification and tracking at earliest and start the cure right away $[19,20]$. As this deadly pathogen is spreading sustainably, easily, and exponentially among mankind, the healthcare workers and medical staff to quell this are severely limited. Due to this scarcity, radiologists are overwhelmed and in severe need of digital tools to take the workload off them. AI experts have suggested a more feasible solution to keep pace in battling this disease by developing ML and DL techniques. Such systems are founded on predicting and diagnosing pneumonia image modalities and scans of the chest thus aiding physicians.

AI-based techniques have shown promising results for various $\mathrm{CV}$ tasks, such as image classification, speech recognition, machine translation, object recognition etc. The recent progress in AI techniques is driven by advent of deeper network architectures, availability of powerful computation platforms and accessibility to large scale benchmark data sets [21]. The DL methods have produced more promising results for various complex CV tasks compared to traditional ML approaches due to their capabilities to learn and represent features automatically. This eliminates the need to manually engineer features based on human expertise and hence obtain higher accuracies for different classification and regression tasks.

Although DL-based methods have been successful in solving various problems, yet they suffer from two main problems: (1) they are extremely difficult to train, and (2) 
Fig. 2 Top 10 most affected countries by COVID-19
TOP 10 AFFECTED COUNTRIES

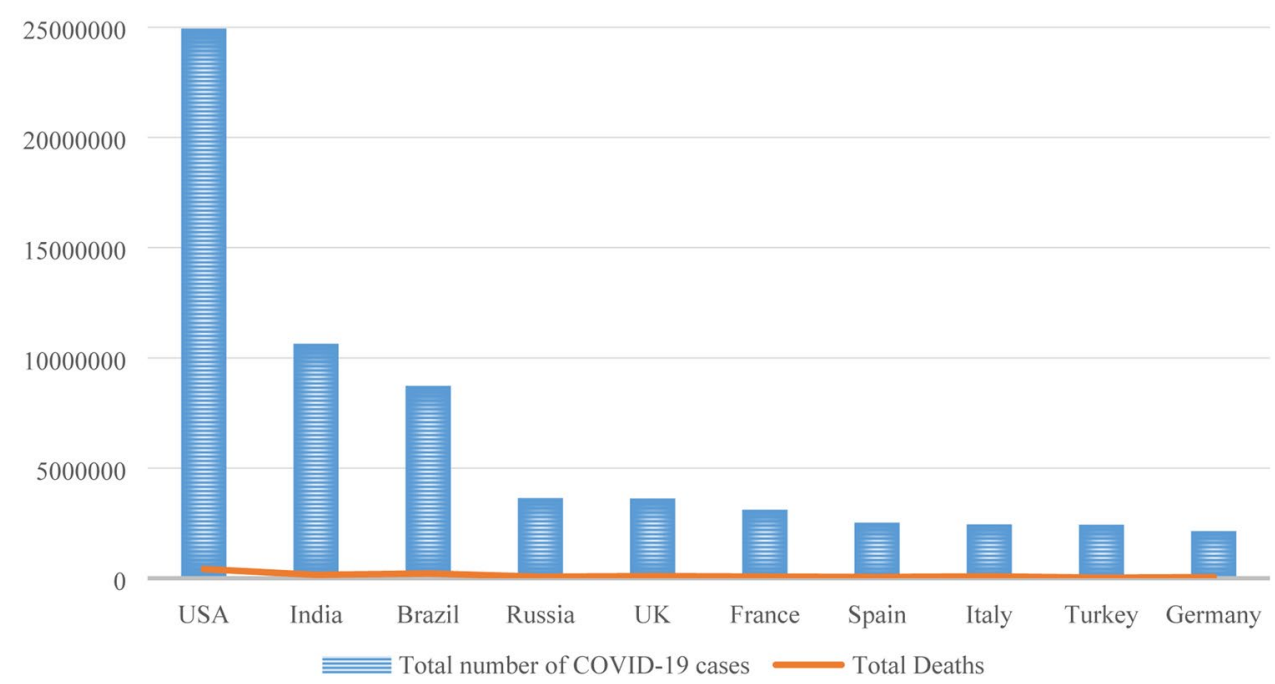

they require large amount of training data. The first problem is usually solved by implementing and running highly optimal code on powerful GPU-based computers. The later problem can also be alleviated by using data generation techniques, such as GANs. The main objective of GAN is to generate additional data that is similar as much as possible to the original training data. This data along with original data is then used to train the DL networks.

Motivated from CV community, the AI methods have also been adopted for medical image analysis.

In addition to the known established procedure to detect COVID-19-infected humans, there is urgency to develop auxiliary tools that can be exploited for identification and monitoring of positive cases. The availability of CT and CXR images of lungs provide certain characteristics linked with COVID-19 [22, 23]. The DL algorithms, such as CNN, incrementally learn the patterns in such images by passing the input data though a sequence of convolutional layers. Initial layers of the network capture low-level features, such as edges, lines, corners etc. while the later layers derive highly abstract features, which can help to capture the most prominent feature that can distinguish between COVID-19 and other cases.

Practicing AI systems for investigation, prediction and analysis of diseases is long-established. The first-ever adoption of such program was fashioned in 1976 called MYCIN which operated and prescribed antibiotics for a bacterial illness [24]. Many healthcare experts have been employing such methods not only to identify diseases but also for formulating drugs, analyzing medical images collected for clinical trials and pandemic prediction.

Many examples of ML and AI medical tools for diagnosis of non-infectious (diabetic, cancer, Parkinson's, heart diseases etc.) [25-29] and contagious diseases (HIV, Ebola,
SARS, and COVID-19) [30-33] were developed. In a recent series, ML methods have been successfully used for Ebola outbreak estimation. The purpose of obtaining a better outcome was achieved by conducting experiments on ten distinctive classifiers giving accuracy results of approximately $90.95 \%$ with $5.39 \%$ MAE and $42.41 \%$ RMSE value [34].

\subsection{Comparisons to Similar Surveys}

The COVID-19 pandemic have turned the center of research activities as scientists and researchers are focusing more to mitigate this disease by proposing various methods in AIbased domain. Meanwhile, experts have presented various review and survey articles based on role of AI in COVID19 to help policy makers and medical practitioners. These peer reviewed published surveys can be categorized into two inculcation; problem-based AI solutions, and AI-frameworks applied on different COVID-19 problems. Such as Pham et al. and Rasheed et al. [35, 36] presented a survey that categorizes the tasks in respond to COVID-19 pandemic by outlining the applications of big data and AI but mostly investigated the papers that have not been peer-reviewed. Moreover, the open research challenges are neither mentioned nor discussed. Similarly, Bansal et al. [37] briefly outlined the role of AI approaches used for identification, prediction and management of COVID-19. However, it did not cover all aspects, such as death rate and severity assessment. In addition, Kumar et al. [38] succinctly generalized the role of DL- and ML-based networks to quell COVID-19 though it did not inspect the papers based on COVID-19 diagnosis through clinical data or respiratory waves. Besides, Lalmuanawma et al. [39] analyzed AI-based applications from various aspects but inspected few papers. 
Contrarily, Hussain et al. [40] overviewed basic AI-based frameworks and Big Data applications applied to combat COVID-19. It elaborated various AI classified learning techniques with cursory details of COVID-19 clinical data analysis and results. Similarly, Swapnarekha et al. [41] categorized the review into type of DL, ML and statistical models to quell COVID-19-related issues. The survey covered vast area, from origin of COVID-19 virus to AI-based models, but focused less on comparative analysis of implemented techniques. A very short survey on COVID-19 detection and prediction is presented in Ref. [42] by analyzing work of only 10 articles.

Beside these, Jamshidi et al. [43] only examined the publication based on advanced DL methods, such as GAN, Extreme Learning Machine, RNN and LSTM for COVID-19 diagnosis and treatment. It just presented the implemented models without comparative analysis. Likewise, Shinde et al. [44] delineates statistical and AI-based forecasting models only, whereas Albahri et al. and Ahmad et al. [45, 46] reviewed only ML and data mining techniques for detection of COVID-19. Similarly, Monshi et al. [47] mainly focused on taxonomy of advanced DL-based methods for generating radiology reports. Some articles reviewed only specific type of data set, such as Jalaber et al. [48] set forth with role of CT images to handle COVID-19 suspected patients at large, severity signs and presentation of lesions, and in the end inspected five articles to describe the role of AI for COVID19 diagnosis. Shaikh et al. [49] investigated AI approaches and landscape of radiographic imaging modalities (CXR, $\mathrm{CT}$ and PET) in few articles with limited information about obtained results. Likewise, Dong et al. [50] mainly highlighted CT and PET-CT imaging characteristics presented in different articles and later compared the AI techniques implemented for COVID-19 detection, while Shi et al. [51] accentuated AI approaches to diagnose COVID-19 that segments CXR and CT images. Articles like Refs. [52, 53] discussed the aspects of IoT and biosensors in COVID-19 management.

The study shows that most of above mentioned review articles either focused on single aspect of COVID-19 management or delineated one type of data set. In addition, majority of these surveys presented little comparative analysis and investigated less than fifty articles, which includes high number of papers that have not been peer-reviewed. Our paper mostly covers peer-reviewed articles that presented AI techniques to accomplish tasks, such as COVID-19 diagnosis, prediction, survival assessment and disease prediction, pandemic outbreak forecasting, and drug discovery. Following are the points, which differentiate this study from aforementioned review and survey papers.

- Covers majority of aspects and problems to manage COVID-19 pandemic, such as diagnosis, prediction, disease severity and survival assessment, outbreak forecasting, protein sequence formation and drug discovery.

- Focuses on both ML- as well DL-based models and frameworks.

- Incorporates all types of data, such as radiographic images (CXR, CT, and ultrasound images), clinical blood samples data, respiratory and coughing waves, time series and other textual data.

- Detail comparative performance analysis of various AIbased techniques implemented to combat COVID-19.

- A comprehensive analysis mostly based on peer-reviewed articles (90\% are peer-reviewed published papers).

\subsection{Scope and Contribution of the Survey}

The primary aim of this comprehensive study revolves around the in-depth analysis of AI-based approaches and models used to quell and combat COVID-19 pandemic by mitigating the virus in various prospects, such as prognosis and diagnosis, drug discovery and molecular structural formation. This review will provide a meaningful and compact knowledge both for medical computer scientist and experts to further broaden the research direction to deal with this deadly virus. The main contributions of this study are as follows:

- The short summary of history, patterns, and characteristics of infectious viruses including COVID-19 are presented.

- AI techniques and tools adopted to mitigate COVID-19 pandemic in various prospects, such as prognosis and diagnosis of SARS-CoV-2 disease, drug discovery and molecular structural formation, are highlighted

- An extensive information regarding approaches to diagnose COVID-19 using radiography images, breathing and coughing wave samples, and clinical blood samples are described in details.

- A comprehensive summary on issues and recommendations to overcome this infectious virus is provided that can timely facilitate effective decision making.

- A detailed discussion on open research challenges regarding COVID-19 is also provided.

The remaining paper is managed as follows. Section 2 focuses on the novel ML and DL techniques that are in practice for diagnosis through various modes. Furthermore, it explores the potential ML- and DL-based tools to predict survival and mortality rate, discover vaccine and forecast the COVID-19 pandemic outbreak. Section 3 presents the issues and recommendation to overcome virus, while Sect. 4 outlines the open research challenges regarding COVID-19 and presents counter-measures to layout a firm groundwork 
for further research. Finally, the conclusion to summarize the overview of this review is presented in Sect. 5.

\section{Al-Based Applications to Quell COVID-19}

The inevitable infectious pandemics are unpredictable and can inflict huge agonies and mortalities across the world. The newly emerged SARS-CoV-2 virus may just be a small capsid but too powerful that requires great efforts and better countermeasures by society to mitigate its negative impact. At the time of this COVID-19-related global emergency, AI researchers had responded the threat by strategically applying various ML and DL techniques in a wide range of applications that not only detect and classify the COVID-19 cases but also forecast the outbreak, tracks the transmission pattern, discovers the effective drugs, predicts the mortality rate and assess the disease's severity.

$\mathrm{AI}$ is a wide-ranging scientific area concerned with mimicking human intellectual processes by smart devices. ML is sub-domain of AI that uses statistical models to learn from examples (also known as instances) in data to predict future outcomes without prior knowledge and explicit programming [54]. Whereas, DL is the most tangible manifestation of ML that exploits artificial neural networks for classification or detection task by discovering useful representations from raw data within the predefined space of possibilities. As COVID-19 pandemic is under the spotlight in medical research and AI-based technologies are one of panacea, this section encompasses various novel applications established on ML and DL methods to combat ongoing SARS-Cov-2 pandemic crisis. Figure 3 unfolds the general approach used to incorporate AI techniques that requires clinical blood samples and radiography images for identification, classification and diagnosis of COVID-19. Various repository are built to store and share data sets regarding COVID-19. Later on, besides data mining, various pre-processing techniques, such as noise removal, data cleaning, feature extraction, segmentation and feature analysis, are mostly employed to enhance the data set and transform it to more meaningful and effective representation. Finally, AI-based techniques and tools are defined to utilize the data sets for COVID-19 segregate COVID-19 affected patients from others.

\subsection{Radiography Image-Based COVID-19 Diagnostic Tools}

Saving precious lives is the topmost priority in emergencies, but that requires early detection of disease. The outbreak of this pandemic created a new landscape and requirements of rapid diagnostic tools for early disease detection. Early ailment detection leads to immediate treatment, which can save many lives and helps in halting the pandemic spread. The standard RT-PCR technique limits the early detection of COVID-19 due to low sensitivity and high procedural and experimental time. Contrarily, AI-based health care systems provide outstanding support for efficient screening, early identification and fast diagnosis by analyzing Clinical blood sample data and radiology images, such as CT and CXR, thus providing a sigh of relief for radiologists.

Researchers and scientists effectively adopted several ML approaches and techniques to curb the COVID-19 ailment, such as Sethy et al. [55], developed automatic tool that predicts COVID-19 infection in CXR images by employing

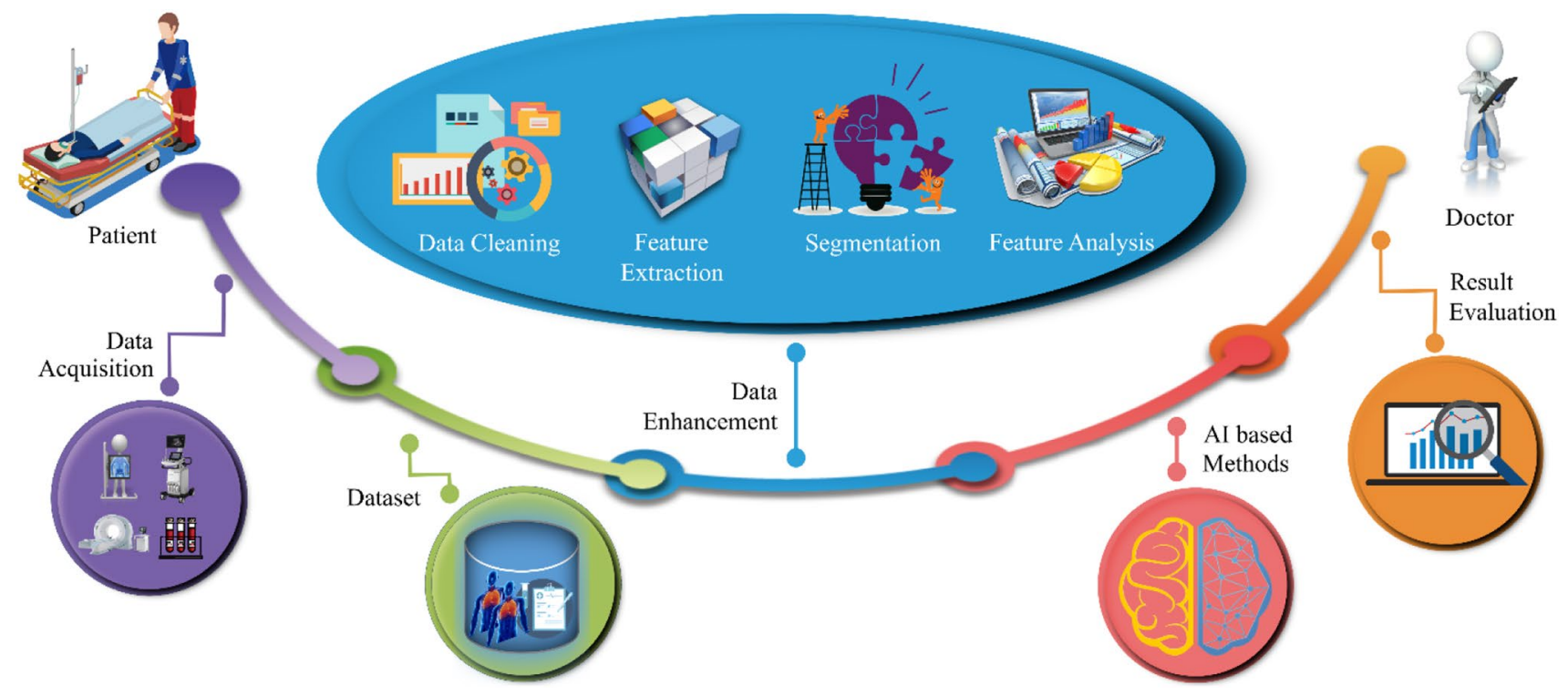

Fig. 3 Illustration of computer vision and AI based model for COVID-19 diagnosis and prediction 
SVM. To segregate the patients affected by COVID-19 among normal and other pneumonia affected patients, they exercised thirteen different pre-trained state-of-the-art models (VGG19, VGG16, AlexNet etc.) to extract features from 381 CXR. Each class/label (normal, COVID-19, bacterial pneumonia) has 127 CXR to balance the data set. Later, SVM classified COVID-19-infected patients by exploiting the extracted deep features. By comparative analysis, author demonstrated that SVM with ResNet50 model achieved 95.33\% average accuracy with a data split ratio of 80:20\% while training and testing, respectively. Moreover, it also accomplished better performance in terms of F1-score and sensitivity of $95.34 \%$ and $95.33 \%$, respectively. A combination of data over-sampling, image augmentation techniques with ML-based classifier has been introduced in Ref. [56]. The researcher first extracted the features by GLCM and its variants, and then used SMOTE for balancing class distribution. The model consists of SVM classifier with stacked AE and PCA to exhibit an accuracy of $94.23 \%$, precision of $96.73 \%$, sensitivity of $91.88 \%$, F1-score of $93.99 \%$, and specificity of $98.54 \%$ on CXR.
An alternative technique has been proposed by Ref. [57] to show the effectiveness of multi-view representation learning that transform original features into latent representation of class space for COVID-19 diagnosis. In pre-processing step, V-Net model [58] extracted pulmonary segments and lung lobes from CT images to segment the infected lesions. They further divided the obtained 189-dimensional features into two radiomic features (Gray, and Texture) using GLCM and its variants, and five handcrafted features (histogram, number, intensity, volume, and surface). The CPM-Nets [59] learnt the later features. Later, they trained Latent-representation Regressor model followed by several ML-based classifier models (LNR, SVM, Gaussian Naïve Bayes, KNN, and logistic regression) for COVID-19 prediction. With the incorporation of proposed model, the model achieved an overall accuracy, specificity and sensitivity of $95.5 \%, 93.2 \%$ and $96.6 \%$, respectively, on $2522 \mathrm{CT}$ images, among which 1495 samples belong to patients affected by COVID-19). Table 1 represents ML-based approaches and classifiers, such as DT, KNN, LNR, and LD used to screen and detect COVID-19 cases by analyzing medical radiology images.

Table 1 Adopting radiography images for COVID-19 diagnostic applications based on machine-learning approaches

\begin{tabular}{|c|c|c|c|c|c|c|c|c|}
\hline Ref & Name of algorithm/model & Problem/assignment & Type of data & Classes & $\mathrm{P}$ & $\mathrm{Sp}$ & $\mathrm{Se}$ & A \\
\hline [55] & $\begin{array}{l}\text { ResNet50 for deep feature extraction and } \\
\text { SVM as classifier }\end{array}$ & COVID-19 detection & CXR & 3 & - & - & 95.3 & 95.3 \\
\hline [56] & $\begin{array}{l}\text { SMOTE for feature oversampling, } \\
\text { stacked Auto-encoders and Principal } \\
\text { Component Analysis for feature extrac- } \\
\text { tion and SVM for classification }\end{array}$ & Classification of COVID-19 & CXR & 6 & 96.7 & 98.5 & 91.8 & 94.2 \\
\hline [57] & $\begin{array}{l}\text { Multi-view representation learning tech- } \\
\text { nique with ML-based classifiers (LNR, } \\
\text { SVM, KNN, NN, and Gaussian Naïve } \\
\text { Bayes) }\end{array}$ & COVID-19 screening & CT & 2 & - & 93.2 & 96.6 & 95.5 \\
\hline [60] & $\begin{array}{l}\text { Adaptive Feature Selection guided Deep } \\
\text { Forest based on Random Forest }\end{array}$ & $\begin{array}{l}\text { Classification of COVID-19 from other } \\
\text { community acquired pneumonia by } \\
\text { extraction location specific features }\end{array}$ & CT & 2 & 93.1 & 89.9 & 93.0 & 91.7 \\
\hline [61] & $\begin{array}{l}\text { Majority voting-based classifier ensemble } \\
\text { of SVM, KNN, Decision Tree, Naïve } \\
\text { Bayes, ANN, and Binary Gray Wolf } \\
\text { Optimization }\end{array}$ & $\begin{array}{l}\text { COVID- } 19 \text { screen by extracting radiomic } \\
\text { texture descriptors }\end{array}$ & CXR & 2 & 99.7 & 99.5 & 99.8 & 99.6 \\
\hline [62] & Decision Tree based on CNN & Detection of COVID-19 & CXR & 2 & 94.0 & 93.0 & 97.0 & 95.0 \\
\hline [63] & $\begin{array}{l}\text { SVM for classification with Social } \\
\text { Mimic Optimization, SqueezeNet and } \\
\text { MobileNetV2 }\end{array}$ & Detection of COVID-19 & CXR & 2 & $98.8^{*}$ & $99.6^{*}$ & $98.3 *$ & $99.2^{*}$ \\
\hline [64] & $\begin{array}{l}\text { Various ML classifiers including kNN, } \\
\text { DT, RF, SVM and MLP with Clus- } \\
\text { HMC }\end{array}$ & $\begin{array}{l}\text { Identifying COVID-19 in multiclass and } \\
\text { hierarchical schemes }\end{array}$ & CXR & 7 & - & 89.0 & - & - \\
\hline [65] & $\begin{array}{l}\text { Five ML classification algorithms with } \\
\text { IRF-based ResExLBP for feature } \\
\text { extraction/selection }\end{array}$ & Diagnosis of COVID-19 & CXR & 2 & - & 100 & 98.8 & 99.6 \\
\hline$[66]$ & $\begin{array}{l}\text { DT, kNN, SVM, kNN, ensemble and } \\
\text { three-naïve Bayes as classifiers }\end{array}$ & Identification of COVID-19 & CT & 2 & 90.6 & 90.3 & 93.5 & 91.9 \\
\hline
\end{tabular}

$P$ precision, $S p$ specificity, $S e$ sensitivity, $F 1$ f1-measure, $A$ accuracy, $C X R$ chest X-ray images, $C T$ computed tomography images

*Values related to classification of COVID-19 class only 
The costly RT-PCR tests kits are short in supply; therefore, AI scientists have proposed various cost-effective solutions by attempting various DL models in prediction, diagnosis and prognosis of SARS-CoV-2 due to outstanding performance in handling and processing complex biological and medical data. Such as Apostolopoulos and Mpesiana [67] exploited transfer learning technique with various state-of-the-art CNN-based frameworks including Inception, Inception ResNet v2, MobileNet v2, VGG19 and Xception to isolate SARS-CoV-2-infected patients among 1427 CXRs images. The analyzing, author concludes that MobileNet v2 surpassed other frameworks by securing sensitivity of $99.10 \%$, specificity of $97.09 \%$, accuracy of $97.04 \%$ on twoclass problem, while on three-class classification task, it achieved an accuracy of $92.85 \%$. Moreover, they tested the implemented models on second data set that contains 224 COVID-19-infected cases images, 714 CXRs of patients with viral pneumonia, and $504 \mathrm{CXRs}$ of healthy person. On this data set, for three-class problem, MobileNet v2 attained accuracy of $94.72 \%$, while for two-class problem, it secured 98.66\% sensitivity, $96.46 \%$ specificity, $96.78 \%$ accuracy. Similarly, Brunese et al. [68] implemented DTL approach with fine-tuned DL-based customized VGG16 framework to differentiate between pulmonary diseases patients and health person (model-1), and then figures out COVID19-infected patients among discovered pulmonary diseases patients (model-2). The suggested model uses $6523 \mathrm{CXRs}$, among which 250 CXRs correspond to COVID-19-infected patients, $2753 \mathrm{CXRs}$ belong to pulmonary diseases patients and 3520 images of healthy patients to diagnose COVID19 while highlighting the potential-infected region due to SARS-CoV-2 virus. Model-1 accomplished a sensitivity, f1-score, specificity, and accuracy, of 96\%, 94\%, 98\%, and $96 \%$, respectively. The experimental finding yields that second model, disease classification model, attained sensitivity, specificity, accuracy and f1-score of $87 \%, 94 \%, 98 \%$, and $89 \%$, respectively. Apart from using the DTL approaches and pre-trained models, authors of Ref. [69] designed and trained a CNN-based network that utilizes features extracted through PCA. The authors further proposed a GAN model to eliminate the class imbalance issue and enhance the data set. The incorporation of PCA not only significantly reduced the computational time but also improved the accuracy to its maximum extent.

Besides CXRs, researchers also focused on CT images, such as Xu et al. [70], designed a 3-D CNN-based framework to isolate patients affected by COVID-19 among healthy and IAVP in timely manner. They segmented 219 COVID-19-infected CT images, 224 IAVP CT images, and 175 normal cases CT images and extracted meaningful features by incorporating ResNet model. Finally, locationattention classification framework achieved an overall prediction accuracy of $86.7 \%$. Jaiswal et al. [71] presented an alternate state-of-the-art CNN-based model to distinguish COVID-19-infected humans using chest CT images. It employed pre-trained DenseNet201 with DTL approach to analyze 1230 CT images of patients other than COVID-19, while 1262 CT images are of SARS-CoV-2 positive cases. The proposed model achieved precision, sensitivity, specificity, accuracy and f1-score of 96.29\%, 96.29\%, 96.21\%, $96.25 \%$, and $96.29 \%$, respectively. Table 2 lists performance details of COVID-19 diagnostic tools and applications based on DL-guided methods that may aid concerned personnel while selecting an appropriate architecture for SARS-CoV2-infected patient's identification.

\subsection{Routine Clinical Data-Based Diagnostic Tools}

Due to expensive radiographic imaging machines, several developing countries and states lacks in CT, CXR and ultrasound machines but has basic blood testing facilities. Keeping in view such scenarios, scientists and programmers have developed AI-based applications and tools to screen COVID-19 positive case using Clinical blood reports. Batista et al. [119] implemented five various ML classifiers, such as RF, NN, LR, SVM, and gradient boosting trees to segregate COVID-19-infected patients by collecting a 235 adult patients blood sample data from hospital in Brazil. The collected data set contains 125 samples of COVID-19 negative patients, while 110 samples belong to COVID19-infected patients. Each data instance had 15 attributes that includes CRP, mean corpuscular hemoglobin, MCV, mean corpuscular hemoglobin concentration, age, gender, hemoglobin, RDW, red blood cells, leukocytes, monocytes, platelets, lymphocytes, basophils, and eosinophils. From experimental findings, it is noted that SVM outperformed other ML approaches by securing a sensitivity, specificity, accuracy, F1-score, NPV, PPV and brier score of $67.7 \%$, $85.0 \%, 84.7 \%, 72.4 \%, 77.3 \%, 77.8 \%$ and $16.0 \%$, respectively, when tested and trained under tenfold cross validation. Table 3 lists COVID-19 diagnostic applications empowered by various AI approaches, which analyzes data related to routine clinical blood samples.

\subsection{Coughing Waves and Respiratory Pattern-Based Diagnostic Tools}

Beside diagnostic applications for COVID-19 based on radiography images or clinical blood samples data, Wang et al. [124] presented a classification network (BI-AT-GRU) which effectively utilizes the respiratory patterns of patients [125]. In addition to the stimulated data, it adequately uses real-world data. The framework discovers and differentiates the respiratory pattern known as Tachypnea (an occurrence of more speedy respiration) among six other patterns of viral infections. Due to scarcity of respiratory data, authors used 


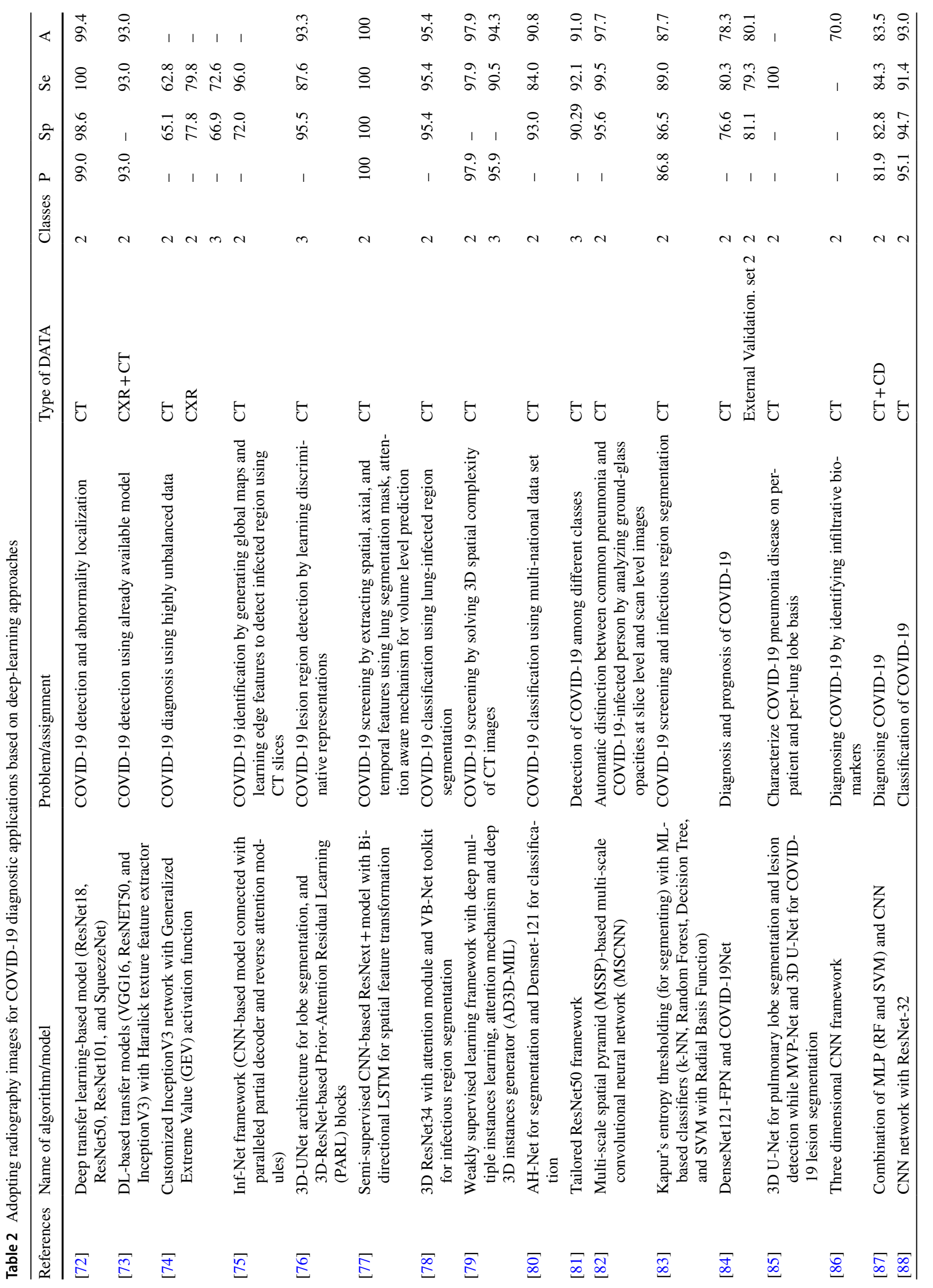




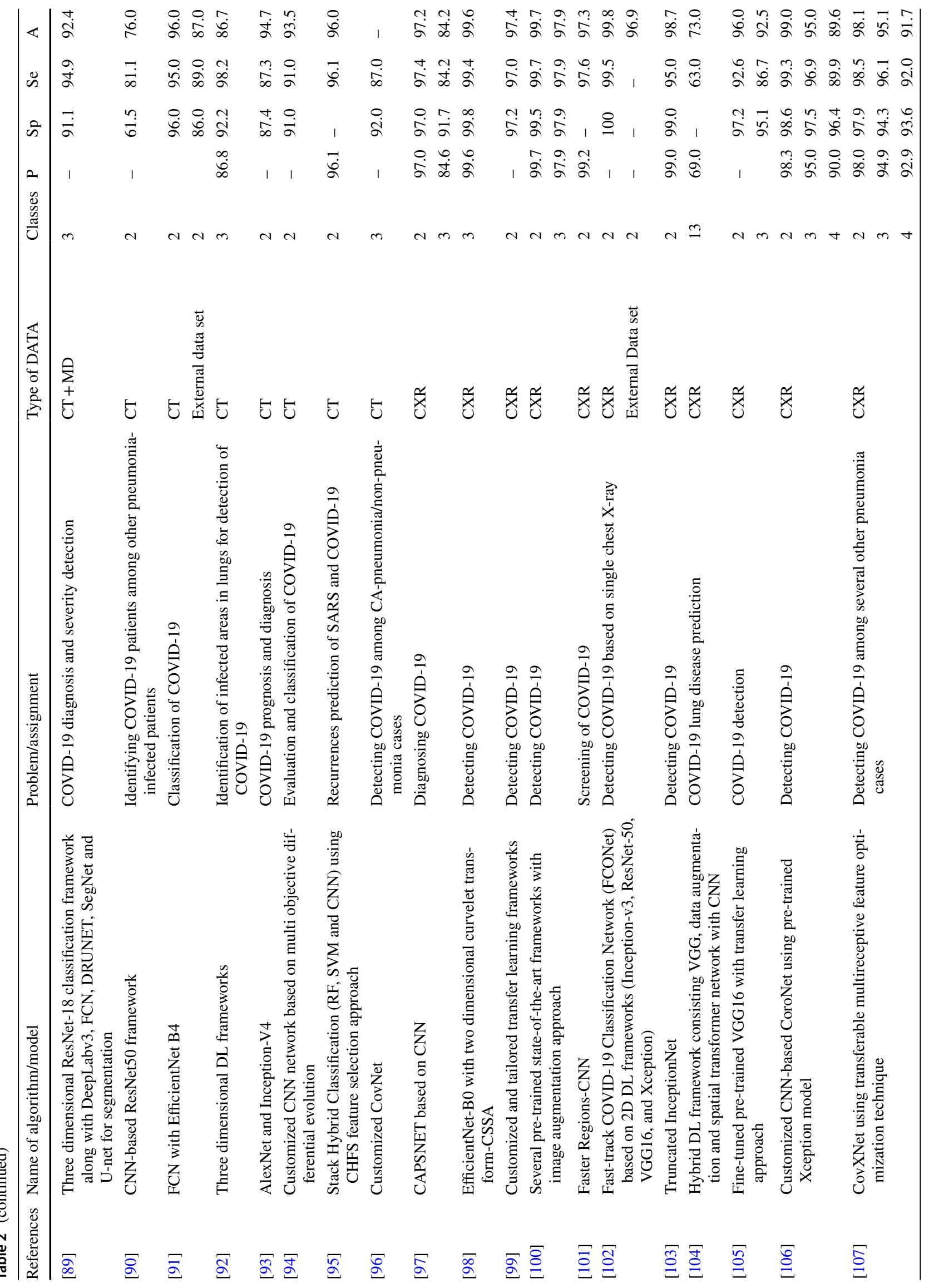




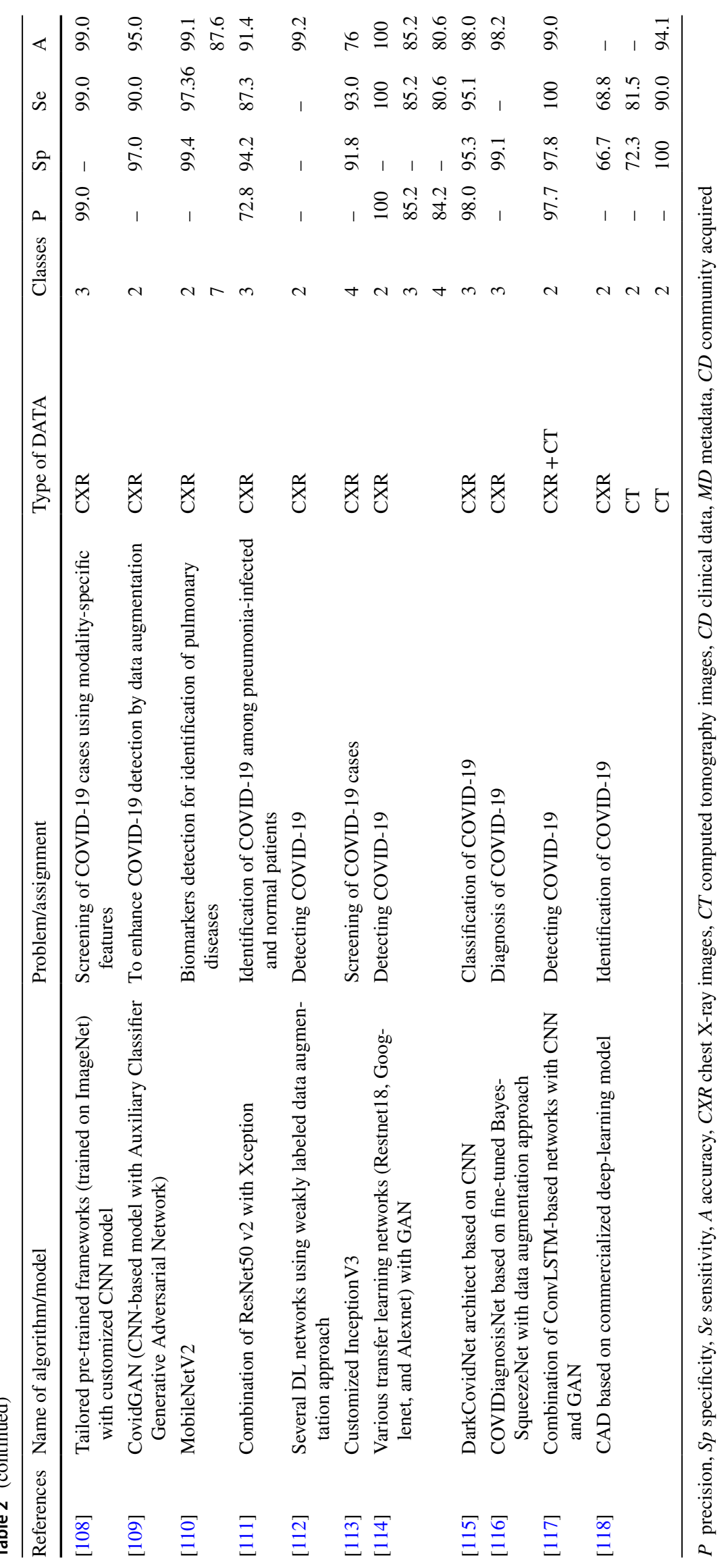


Table 3 Artificial intelligence-based diagnostic tools for COVID-19 using data related to clinical blood samples

\begin{tabular}{|c|c|c|c|c|c|c|c|c|}
\hline References & Name of algorithm/model & Problem/assignment & Type of data & Classes & $\mathrm{P}$ & $\mathrm{Sp}$ & $\mathrm{Se}$ & A \\
\hline$[120]$ & $\begin{array}{l}\text { Several machine learning classifiers } \\
\text { (DT, SVM, kNN, RF, LR, and Naïve } \\
\text { Bayes) }\end{array}$ & $\begin{array}{l}\text { Diagnosis of COVID-19 diagnosis using } \\
\text { hemato-chemical values obtained from } \\
\text { blood examination }\end{array}$ & Text & 2 & 83.0 & 65.0 & 92.0 & 82.0 \\
\hline$[121]$ & $\begin{array}{l}\text { Various machine learning-based models } \\
\text { (LR, RF, DT, and Gradient-boosted } \\
\text { DT) }\end{array}$ & $\begin{array}{l}\text { Diagnosing COVD-19 by considering } \\
\text { regular laboratory tests }\end{array}$ & Text & 2 & - & 80.8 & 76.1 & - \\
\hline [122] & $\begin{array}{l}\text { Feature engineering using TF-IDF with } \\
\text { seven different supervised machine } \\
\text { learning classifiers (DT, Stochastic } \\
\text { Gradient Boosting, LR, RF, Adaboost, } \\
\text { SVM, and Multinomial Naïve Bayes) }\end{array}$ & $\begin{array}{l}\text { Classifying COVID-19 cases among } \\
\text { various viral pneumonia with the use } \\
\text { of use of clinical reports }\end{array}$ & Text & 4 & 94.0 & - & 96.0 & 96.2 \\
\hline [119] & LR, RF, and SVM & COVID-19 diagnosis & Text & 2 & 77.8 & 85.0 & 67.7 & 84.7 \\
\hline$[123]$ & Random Forest & $\begin{array}{l}\text { Identifying COVID- } 19 \text { cases by con- } \\
\text { sidering } 49 \text { different parameters of } \\
\text { clinical data }\end{array}$ & Text & 4 & & 96.9 & 95.1 & 95.9 \\
\hline
\end{tabular}

$P$ precision, $S p$ specificity, Se sensitivity, $A$ accuracy

RSM to generate stimulated breathing patterns. From discussion, it concludes that BI-AT-GRU framework outperformed other state-of-the-art models, such as GRU, LSTM, and BI-AT-LSTM by accomplishing a precision of $94.4 \%$, recall of $95.1 \%$, accuracy of $94.5 \%$, and f1-measure of $94.8 \%$ when tested on real-world data obtained from depth camera. Table 4 illustrates various ML- and DL-based COVID-19 diagnostic tools and applications that uses coughing or respiratory data.

\subsection{Disease Severity and Survival-Mortality Assessment Models}

The timely knowledge about the severity of disease facilitates the attending staff in dealing patients priority wise and utilizing the hospital facilities accordingly. A very rare work has been observed on lung's ultrasound data, such as Carrer et al. [129], proposed a unsupervised method based on Viterbi algorithm and Hidden Markov model for localization and detection of pleural line in LUS data. Later it evaluates the severity level of patient using SVM. From results, it is observed that pleura detection model achieved an accuracy of $94 \%$ and $84 \%$ for linear and convex probes, while SVM classifier evaluated the severity with an accuracy of $94 \%$ and $88 \%$ for linear and convex probes. Zhou et al. [130] proposed machine-agnostic quantification and segmentation technique to cater $3 \mathrm{D}$ segmentation problem in $\mathrm{CT}$ images for severity identification of COVID-19-infected regions. The proposed simulator decreases the model parameters by using symmetry properties of lungs and tissues that decomposes 3D segmentation into three 2D ones (along $y-z, x-y$, and $x-z$ planes) thus reduces time complexity. The three independent 2D U-Nets segmented infectious

Table 4 Artificial intelligence-based diagnostic tools for COVID-19 using respiratory data

\begin{tabular}{|c|c|c|c|c|c|c|c|c|}
\hline References & Name of algorithm/model & Problem/assignment & Data-type/modality & Classes & $\mathrm{P}$ & $\mathrm{Sp}$ & $\mathrm{Se}$ & A \\
\hline \multirow[t]{2}{*}[126]{} & \multirow{2}{*}{$\begin{array}{l}\text { Deep Transfer-Learning- } \\
\text { based Multiclass classi- } \\
\text { fier (DTL-MC) }\end{array}$} & \multirow{2}{*}{$\begin{array}{l}\text { Analyzing irregularities of } \\
\text { pathomorphological muta- } \\
\text { tion in respiratory process to } \\
\text { diagnosis COVID-19 }\end{array}$} & \multirow[t]{2}{*}{ Sound waves/coughing } & 2 & 91.4 & 91.1 & 94.5 & 92.8 \\
\hline & & & & 4 & $89.9 *$ & $96.6^{*}$ & $89.1 *$ & $92.6^{*}$ \\
\hline$[127]$ & $\begin{array}{l}\text { Feature extraction using } \\
\text { VGGish with LR and } \\
\text { SVM for classification }\end{array}$ & $\begin{array}{l}\text { Analyzing coughing samples to } \\
\text { diagnose COVID-19 }\end{array}$ & $\begin{array}{l}\text { Sound waves/coughing and } \\
\text { breathing }\end{array}$ & 2 & 80.0 & - & 72.0 & - \\
\hline$[128]$ & Bi-AT-GRU & $\begin{array}{l}\text { Identifying COVID-19 cases } \\
\text { by examining RBG and ther- } \\
\text { mal videos }\end{array}$ & Thermal videos/breathing & 2 & - & 76.3 & 90.2 & 83.6 \\
\hline$[124]$ & BI-AT-GRU & $\begin{array}{l}\text { Detecting positive COVID-19 } \\
\text { cases }\end{array}$ & Patterns of breathing & 2 & 94.4 & - & 95.1 & 94.5 \\
\hline
\end{tabular}

$P$ precision, $S p$ specificity, $S e$ sensitivity, $A$ accuracy

*Values related to classification of COVID-19 class only 
regions along three orthogonal directions and later integrated these entire masks together with Mask R-CNN to produce final segmentation. The tool achieved an average dice of $76.4 \%, 82.3 \%$ and $87.5 \%$ on early, progressive and severe cases for segmentation problem, while it attains a 96.7\% Pearson correlation coefficient for quantification task.

Bai et al. [131] proposed a hybrid DL-based network with multivariate LR classifier to predict COVID-19 malignant progression. The model converts statistical instances ( 75 clinical data characteristics) to 40-D feature vector using MLP. Finally, it predicts high-risk patients using quantitative CT sequences, obtained at different time interval, along with these transformed multi-dimensional feature vectors with the help of LSTM. The proposed severity assessment tool secured an AUC of $95.4 \%$ and overall accuracy of $89.1 \%$ when evaluated on data set of 133 patients under fivefold cross-validation. Similarly, an ML-based MCDM has been proposed in Ref. [132] to optimize the treatment strategy. The network detects the severely infected SARS-CoV-2 patients and prioritized them for relevant convalescent plasma transfusion. Table 5 shows the severity and fatality assessment models based on conventional ML and advanced DL techniques.

\subsection{COVID-19 Outbreak Forecasting Models}

The widespread of COVID-19 outbreak has created panic as a human community is still at risk, while hospitals are full, people are facing financial issues as governments are struggling to pass critical decisions, mortality rate is increasing exponentially, whereas social activities are halted. In this high uncertainty, experts have applied various DL and ML techniques to design outbreak-forecasting models that would help decision makers to recommend new preventive strategies and develop critical measures for future possibilities. Such as Carrillo-Larco and Castillo-Cara [144] suggested an unsupervised ML-based model that uses $k$-means clustering algorithm to classify the countries sharing same number of confirmed COVID-19 cases. In this study, researchers considered different attributes, such as prevalence of tuberculosis and HIV/AIDS diseases in 156 countries, social-economic parameters, such as gross domestic production as social-economic parameters and other health system metric, such as air quality along with COVID-19 prevalence data (confirmed cases, death etc.). It concludes that the integration of PCA with k-means-based model successfully stratify countries into five and six groups. The researchers conclude that model works well for countries stratification based on confirmed SARS-CoV-2 cases but not able to classify in terms of SARS-CoV-2 fatality cases.

Table 5 Mortality and survival rate prediction with disease severity assessment of patients using artificial intelligence-based application

\begin{tabular}{|c|c|c|c|}
\hline References & Name of algorithm/model & Problem/assignment & Type of data \\
\hline [133] & $\begin{array}{l}2 \text { stage 3D U-Net for lobe segmentation and } \\
\text { 3D-inflated modified variant of Inception for } \\
\text { COVID-19 Reporting and Data System (CO- } \\
\text { RADS) score prediction }\end{array}$ & $\begin{array}{l}\text { Severity assessment of COVID-19-infected patients } \\
\text { by automatic segmentation of pulmonary lobes of } \\
\text { lung }\end{array}$ & $\mathrm{CT}$ \\
\hline [134] & 3D CNN-based network with VB-Net & COVID-19 quantification and detection & $\mathrm{CT}$ \\
\hline [135] & Deep neural network based on six dense layers & $\begin{array}{l}\text { Mortality prediction in COVID-19 patients using } \\
\text { clinical data }\end{array}$ & Text \\
\hline [136] & VGG16 & $\begin{array}{l}\text { Analyzing and assessing severity of COVID-19 infec- } \\
\text { tion in lungs }\end{array}$ & Radiography images \\
\hline [137] & $\begin{array}{l}\text { Various machine learning classifiers (Elastic Net, RF, } \\
\text { Adaboost Pregressor, DT, SVM, and Huber Regres- } \\
\text { sion etc.) }\end{array}$ & $\begin{array}{l}\text { Analyzing COVID- } 19 \text { transmission by examining the } \\
\text { humidity and atmospheric temperature }\end{array}$ & Textual and TS \\
\hline [138] & $\begin{array}{l}\text { Customized CNN framework with fractal techniques } \\
\text { for feature extraction }\end{array}$ & Assessing COVID-19 disease severity & CXR \\
\hline [139] & DenseNet model & $\begin{array}{l}\text { To find the severity of COVID-19 lung and the } \\
\text { degree of opacity in lung }\end{array}$ & CXR \\
\hline [140] & Fine-tuned RF with AdaBoost & $\begin{array}{l}\text { Predicting disease severity to highlight chances of } \\
\text { death or recovery }\end{array}$ & Text \\
\hline [141] & Various LNR models & $\begin{array}{l}\text { Diagnosing COVID-19 cases and predicting its } \\
\text { volume and severity }\end{array}$ & $\mathrm{CT}$ and $\mathrm{CD}$ \\
\hline [142] & SVM & Predicting recovery cases & Text \\
\hline [143] & SVM & $\begin{array}{l}\text { Critical cases detection among patients with mild } \\
\text { symptom }\end{array}$ & Text \\
\hline
\end{tabular}

$C X R$ chest X-ray images, $C T$ computed tomography images, $C D$ clinical data, $T S$ time series 
To assist policymaker, Kavadi et al. [145] presented a PDR-NML framework that predicts SARS-CoV-2 transmission patterns in India. The proposed statistical model, PPDLR, normalizes it by searching the best features, which are then fed to a support Kuhn-Tucker-based nonlinear global pandemic ML model to forecast the future outbreak of COVID-19 pandemic cases. The presented model outperformed LNR and other famous AI-base models by securing 99.7\% accuracy. Hu et al. [146] attempted modified AE that uses real-time data for pandemic outbreak forecasting in large geographical area by considering the interventions and measures to curb the pandemic. The framework use data of confirmed COVID-19 cases happened between January 20, 2020 to March 16, 2020 in 152 countries to forecast future cases, as well as pandemic peak and end time. It consists of 2 single AEs each comprised of 3 feed-forward NN layers that performed well while estimating the daily new cases in China as compared to SEIR model. The model attained error of 0.00134 and recommended that early precautionary measures would eliminate $99.4 \%$ of potential cases.
Other than the conventional ML algorithm, experts also used advanced DL techniques to determine the unseen forthcoming cases. Authors in Ref. [147] forecasted global pandemic outbreak by using multivariate spatiotemporal model based on convolutional LSTM framework. They used the data of Italy and USA, and transformed spatial features into clusters. The proposed forecasting tool predicted number of potential cases for the next 5 days with an MAPE of 5.57\% and $0.3 \%$ for USA and Italy, respectively. Table 6 illustrates more AI-based applications/tools to assess the risk and predict the pandemic outbreak.

\subsection{COVID-19 Protein Sequence Formation and Drug Discovery Models}

In this panic-stricken era of COVID-19, rapid drug discovery in accordance with the exact virus genome is crucial to saving thousands of lives. Still many genomes and peptides of this noxious virus are being identified on a regular basis. To bring the effective drug-making process up to speed,

Table 6 COVID-19 outbreak prediction and risk assessment using artificial intelligence-based applications

\begin{tabular}{|c|c|c|c|}
\hline References & Name of algorithm/model & Problem/assignment & Type of data \\
\hline [148] & Several ML-based classifiers (DT, LR, SVM and RF) & Forecast COVID-19 spreading patterns in 42 countries & Text \\
\hline [149] & ARIMA, Bi-LSTM, LSTM, and SVM & $\begin{array}{l}\text { Forecast death, recovery rate and potential cases in major } \\
\text { countries }\end{array}$ & TS \\
\hline$[150]$ & ARIMA and Least square-SVM & Estimate COVID-19 cases for the next month & TS \\
\hline [151] & RNN, Bi-LSTM, LSTM, GRU, LSTM, and VAE & $\begin{array}{l}\text { Predict (on short term) the new contaminated and recov- } \\
\text { ered patients }\end{array}$ & TS \\
\hline [152] & FbProphet & COVID-19 epidemic trend prediction & TS \\
\hline$[1]$ & ANN-based adaptive incremental network & $\begin{array}{l}\text { Monitor and analyze the disease's growth stimulation for } \\
\text { forecasting and population Compartmentalization based } \\
\text { on its risk }\end{array}$ & TS \\
\hline [153] & Polynomial Regression. LNR, and SVM & Predict the migration type, growth and transmission rate & Text \\
\hline$[154]$ & LNR, MLP and Vector autoregression method & COVID-19 spread prediction in India & TS \\
\hline$[155]$ & $\begin{array}{l}\text { Various ML-based models (SVM, LNR, Exponential } \\
\text { Smoothing, and Least Absolute Shrinkage and Selec- } \\
\text { tion Operator) }\end{array}$ & $\begin{array}{l}\text { Forecast cases, deaths and recoveries due/from COVID- } \\
19 \text { in the next } 10 \text { days }\end{array}$ & TS \\
\hline [156] & Unsupervised-SOM & $\begin{array}{l}\text { Spatially cluster the countries having similar COVID-19 } \\
\text { cases }\end{array}$ & TS \\
\hline [157] & Cloud computing with ML-based approach & $\begin{array}{l}\text { Predict the growth and analyze potential threat related to } \\
\text { COVID- } 19\end{array}$ & TS \\
\hline [158] & LSTM with LNR & Forecast COVID-19 outbreak trends in Iran & TS \\
\hline [159] & Wavelet transform approach with Regression tree & $\begin{array}{l}\text { COVID-19 outbreak prediction/forecasting in various } \\
\text { countries and assessing the risk }\end{array}$ & TS \\
\hline$[160]$ & Fuzzy rule induction with Composite Monte Carlo & Future possibilities prediction & TS \\
\hline$[161]$ & LSTM with Curve fitting & $\begin{array}{l}\text { Analyze the effect of social distancing and lockdown on } \\
\text { predicting COVID-19 cases }\end{array}$ & TS \\
\hline$[162]$ & SEIR & $\begin{array}{l}\text { Examine the effect of control measures while predicting } \\
\text { COVID-19 outbreak }\end{array}$ & TS \\
\hline [163] & Customized SEIR with LSTM & $\begin{array}{l}\text { Analyze and predict COVID-19 pandemic curve for } \\
\text { China }\end{array}$ & TS \\
\hline
\end{tabular}

$T S$ time series 
many ML models are in process to master the viral structural analysis. In the interest of seeking probable vaccine possibilities for SARS-CoV-2, Ong et al. [164] established a machine learning-based Vaxign-ML reverse vaccinology system. Their research based on a system equipped with five conventional ML algorithms (XGB, SVM, RF, kNN and logistic regression) applied on extracted protein data set, subsequent to fivefold cross-validation, defined further with biological and physicochemical characteristics,. From findings, it can be noted that XGB model indicated an F1-measure of $94 \%$. The results of the system indicated conservancy of SARS-CoV-2 N protein sequence with SARS$\mathrm{CoV}$ and MERS-CoV only. While discussing the crucial matters of virus attacking and attaching itself to the host, the responsible adhesions proteins found were the $\mathrm{S}$ protein along with non-structural proteins; nsp3, 3CL-pro, and nsp810. Moreover, high protective antigenicity causing inferred by the designed Vaxign-ML system was attributed to three proteins namely S, nsp3, and nsp8 as potential vaccine candidate based on high protegenicity score. This particularly tailored vaccine aptitudes for designing a reliable and competent COVID-19 vaccine.

Magar et al. [165] founded a machine learning idea envisioned to identify synthetic COVID-19 inhibitory antibodies. The ML strategies segregated the data of virus-antibody sequences via graphical diagrams and reported 8 stable antibodies with the ability to perform as COVID-19 inhibitors. The way that led the COVID-19 antibody identification starts with gathering and maintaining the data for the devised system. Then comes the featurization, embedding and benchmarking ML designs and screening for the best model available. Later on, a hypothetical antibody group is assembled and ML screening is done for obliteration. Lastly, the validity of the suggested antibodies is evaluated. Such generalized flowchart makes fast and facile screening of probable antibodies having an immense potential to defeat COVID-19. Table 7 depicts other AI-empowered applications used to detect protein sequence and discover to tackle COVID-19 pandemic.

\section{Discussion}

In this survey, we not only presented an analysis of clinically utilized AI tools providing assistance against COVID19 but also presented a detailed historical account of the virus and related family. The detailed investigation reveals several ML and DL approaches so far lending help to a great extent, initiating from image diagnostics and going up to the presentation of prospective models for methodical anticipation of the epidemics outbreaks. Therefore, this study can facilitate healthcare and research operatives to efficiently and effectively cope the COVID-19 pandemic. With timely and precise analysis, approach at hand for the desired solution an immediate response against the disease can be lead. This survey concentrates mainly on the obstacles faced so far while executing AI and ML-based arrangements for COVID-19 prognosis. Moreover, some suggested plans and quick fixes to resolve those issues are also covered.

The foremost inaccuracy fails to arise from not constituting diagnostic programs, which segregates on the basis of symptoms. Thus, to have robust and well-generalized predicting tools, various other aliments (liver, heart, diabetes, etc.) and patient's information (gender, age, etc.) must be considered. In comparison with known community acquired and viral pneumonia, these aspects (patient's information and aliments) have significantly influences the severity of COVID-19 disease in a human. Thus, leading to an instantaneous identification of the virus. An enormous amount of data is laid out there by several countries severely hit by the pandemic. If those statistics are attained in the right way, schemes for the prediction can efficiently generate smart and uncomplicated results.

Then, there comes the issue of urgency. Since the government needs statistics right away to regulate quarantine laws for constraining the disease expansion. Therefore, the scientific data sets are constructed on hasty calculations. Thus, providing ineffective and non-reproducible structures. In pursuance of productive and quick implementation of policy models, the data set must be fixed and generate reproducible

Table 7 Protein sequence detection and drug discovery using artificial intelligence-based applications

\begin{tabular}{lll}
\hline References & Name of algorithm/model & Problem/assignment \\
\hline$[164]$ & DTL-based Vaxign-machine-learning reverse vaccinology tool & Candidate vaccine prediction for SARS-CoV-2 virus \\
{$[166]$} & LSTM and semi-supervised VAE & Discover drug by detecting SMILE fingerprint of molecules \\
{$[167]$} & GAN & Designing the drug compound (non-CoV) \\
{$[168]$} & A pre-trained network based on AI approach, called Molecule- & Determine the availability of antiviral drug to tackle SARS-COV-2 \\
& Transformer-Drug-Target-Interaction & \\
{$[165]$} & SVM, RF, MLP, LR, and XGBoost & Potential antibodies discovery for COVID-19 \\
{$[169]$} & MLP and ANFIS & Detect nucleic acid based on CRISPR \\
{$[170]$} & GAN & Develop formation of drug compound for COVID-19 \\
\hline
\end{tabular}


outcomes and solve the urgent problem as well. In designing the analysis applications based on statistical approach, one of the most important and challenging task is availability of high-quality samples at real time. In circumstances, where data are rapidly changing at faster pace, the reliability of produced tools are minimized. Therefore, to tackle this problem, regular updating the data based on region-specific values are decisive.

For planning any systems for prediction studies, attaining large data is the crucial requirement that needs to be fulfilled. Deep learning-based systemization needs big data sets of clinical images and statistical numerals. A large amount of data can be produced for better training of deep learning approaches if obtained sets are not divided on the basis of geography. Regulation of data sources needs to be addressed as well while handling massive data to avoid misunderstandings. Yet, another challenge that arises while training and designing such AI systems is due to little or almost no association of researchers with medical experts. While assessing the medical data, such as X-ray or scans relatable medical personnel, must be present to make a definitive opinion.

A better and definitive estimation of potential patients and the number of deaths that are going to happen in future is a top priority in the current situation. This can be achieved by gathering and structuring the data of heavily affected countries and utilizing it with accurate and robust AI-based models as better equipment against an upcoming alarming situation.

\section{Open Research Challenges}

The COVID-19 pandemic has unequivocally affected both individuals and economies everywhere in the world. This led to various systemic challenges in various field, such as health, governance, trade, education, technology etc. This section highlights some of the challenges to combat the current pandemic.

\subsection{Research Collaboration and Data Sharing}

The COVID-19 is a family of viruses that has various forms and has different behavior on people living in various regions around the globe. This requires a cross examination of the cases in multi-folded perspectives. There must be some agreements reached among various entities including civil society, private and public sectors to share data and conduct research to expedite the process for finding an ultimate solution. This envisions the authorities about expected abiding transformations, and motivates them to revamp the condition of the world by taking advantages of this moments.
It is critical for combating the disease to do research collaboration to accelerate the process towards normalizing the life and overcome the pandemic. This requires high-level decision making to remove obstacles in free flow of research ideas and make availability of research data for more critical decision making. Infrastructure should be developed to share and collaborate the research data globally, which is still a challenging task. This also requires policy to be defined for integrity of the patient data by introducing some defined set of rules and measures, interoperability and co-ordination, in conjunction with quality and interpretation of data.

\subsection{Lack of Technology Infrastructure}

Availability of IT infrastructure is crucial for early detection, tracking and monitoring of the patients. In China, ubiquitous availability of IT infrastructure help finding hotspots and crowd gathering, thus assisted the administrators to take faster decision making and implementation. Developing countries are suffering from serious lack of infrastructures to fully utilize the ability of AI for both detection and previous strategies.

\subsection{Development of Vaccines}

AI is inevitable in almost all fields of life. Multidisciplinary research should be conducted for development of vaccine for COVID-19. The use of AI for drug delivery, design, development and efficacy will eventually speed up the drug testing in real time. Policies should be designed to take advantage of this.

\subsection{Increased Pressure on Hospitals}

There has been sharp increase of patient admissions in hospitals across the globe that overwhelmed the healthcare professionals due to high workload. To increase the efficiency and properly managing the critical patients, AI should be used from patients' admission, testing and monitoring. This will reduce the pressure and workload for medical staff.

\subsection{Adopting Models from Developed Countries}

Some countries, such as South Korea, flattened the curve of COVID-19 by adopting digital technology and combining it with other strategies, such as early detection, free treatment, and isolation of COVID-19 cases. No severe lockdowns were placed; however, they used technology to monitor trends and hotspots to take early action. In addition, the public awareness and participation was very good. Adopting such model in the developing countries need these three important components: digital infrastructure, healthcare infrastructure, and public awareness. 


\subsection{Transparent Disclosing all Information}

Accurate information sharing is crucial for public safety and crucial decision making. Governments should develop strategies for sharing the accurate information about the COVID19 cases, through press briefing, to make awareness among mankind about ways to minimize the transmission of virus, and the impact of the social distancing.

Finally, the developing countries must cautiously and gently relaxes the restrictions related to immigration and quarantine keeping in view the global transmission rate of disease and corresponding healthcare situation/facilities of the country. Countries should also aim for assistance and capacity building in communal institutions. International associations and societies must collaborate and join the developing countries to cope with this pandemic.

\subsection{Lesson Learning from this Pandemic}

This may not be the last pandemic, as world has witnessed many similar challenges in the past. Learning some lessons from current pandemics and preparing for future challenges is a crucial step now. The models and preparedness should be done keeping future perspective in mind. Creating awareness among people and balanced distribution of wealth can highly reduce the risks of spread of such diseases.

\section{Conclusion}

The recent advancements in AI techniques have played an important role in biomedical sciences providing a handy role in diagnosis and monitoring of various diseases. For COVID-19 pandemic, it is essential to detect it as early as possible by collecting and analyzing related information to predict, where this virus will affect in the future. In this paper, we report the recent active role of AI for combating the COVID-19 and highlighted new research directions and main challenges in adopting a robust solution for this pandemic. The paper spans over two main domains, medical and technological. In the first domain, this review covered the global transmission patterns of COVID-19 and brief history of various other viruses. Then, it described the advances in AI tools to diagnose, assess the severity of disease, predict the mortality rate, and discover the drug compounds. Furthermore, an analysis of recently applied techniques for various biological and computing approaches have been presented. This work will help decision makers to better understand the role of AI for combating COVID-19, so that better decisions can be taken to properly handle and take precautionary steps by designing instructions in pandemic stricken regions. Subsequently, computer-aided platforms are in operation for smart utilization of medical facilities on a priority basis.

\section{Declarations}

Conflict of interest The authors declare that they have no conflict of interest. The authors declare that they have no known competing financial interests or personal relationships that could have appeared to influence the work reported in this paper.

Informed Consent For this type of study, formal consent is not required.

Statement of Human and Animal Rights This paper does not contain any studies with human participants or animals performed by any of the authors.

\section{References}

1. Farooq J, Bazaz MA (2020) A novel adaptive deep learning model of COVID-19 with focus on mortality reduction strategies. Chaos Solitons Fractals 138:110148. https://doi.org/10.1016/j. chaos.2020.110148

2. Cui J, Li F, Shi ZL (2019) Origin and evolution of pathogenic coronaviruses. Nat Rev Microbiol 17:181-192. https://doi.org/ 10.1038/s41579-018-0118-9

3. Barquet N, Domingo P (1997) Smallpox: the triumph over the most terrible of the ministers of death. Ann Intern Med 127:635642. https://doi.org/10.7326/0003-4819-127-8_part_1-19971 0150-00010

4. Mahadevan A, Suja MS, Mani RS, Shankar SK (2016) Perspectives in diagnosis and treatment of rabies viral encephalitis: insights from pathogenesis. Neurotherapeutics 13:477-492. https://doi.org/10.1007/s13311-016-0452-4

5. Orbann C, Sattenspiel L, Miller E, Dimka J (2017) Defining epidemics in computer simulation models: How do definitions influence conclusions? Epidemics 19:24-32. https://doi.org/10. 1016/j.epidem.2016.12.001

6. Centers for Disease Control and Prevention (CDC): Principles of Epidemiology in Public Health Practice (2006)

7. Henderson DA (2009) Smallpox-the death of a disease. Prometheus Books, New York

8. Gorbalenya AE, Baker SC, Baric RS, de Groot RJ, Drosten C, Gulyaeva AA, Haagmans BL, Lauber C, Leontovich AM, Neuman BW, Penzar D, Perlman S, Poon LLM, Samborskiy DV, Sidorov IA, Sola I, Ziebuhr J (2020) The species severe acute respiratory syndrome-related coronavirus: classifying 2019-nCoV and naming it SARS-CoV-2. Nat Microbiol 5:536-544. https:// doi.org/10.1038/s41564-020-0695-Z

9. Zaki AM, van Boheemen S, Bestebroer TM, Osterhaus ADME, Fouchier RAM (2012) Isolation of a novel coronavirus from a man with pneumonia in Saudi Arabia. N Engl J Med 367:18141820. https://doi.org/10.1056/NEJMoa1211721

10. Zhu N, Zhang D, Wang W, Li X, Yang B, Song J, Zhao X, Huang B, Shi W, Lu R, Niu P, Zhan F, Ma X, Wang D, Xu W, Wu G, Gao GF, Tan W (2020) A novel coronavirus from patients with pneumonia in China, 2019. N Engl J Med 382:727-733. https:// doi.org/10.1056/NEJMoa2001017

11. England NHS, Henderson K, Medicine E, England NHS, Uk A, Nhs A, England NHS, Medicine A, Nhs T, Pharmacist C, 
Service C (2020) Seven days in medicine: 8-14 Jan 2020. BMJ 368:m132. https://doi.org/10.1136/bmj.m132

12. Moriyama M, Hugentobler WJ, Iwasaki A (2020) Seasonality of respiratory viral infections. Ann Rev Virol 7:1-19. https://doi. org/10.1146/annurev-virology-012420-022445

13. Rothan HA, Byrareddy SN (2020) The epidemiology and pathogenesis of coronavirus disease (COVID-19) outbreak. J Autoimmun 109:102433. https://doi.org/10.1016/j.jaut.2020.102433

14. Zhou F, Yu T, Du R, Fan G, Liu Y, Liu Z, Xiang J, Wang Y, Song B, Gu X, Guan L, Wei Y, Li H, Wu X, Xu J, Tu S, Zhang Y, Chen H, Cao B (2020) Clinical course and risk factors for mortality of adult inpatients with COVID-19 in Wuhan, China: a retrospective cohort study. Lancet 395:1054-1062. https://doi. org/10.1016/S0140-6736(20)30566-3

15. WHO: Coronavirus (COVID-19) events as they happen (2020) https://www.who.int/emergencies/diseases/novel-coronavirus2019/events-as-they-happen

16. Wang L, Gao Y-H, Lou L-L, Zhang G-J (2020) The clinical dynamics of 18 cases of COVID-19 outside of Wuhan, China. Eur Respir J 55:2000398. https://doi.org/10.1183/13993003. 00398-2020

17. Worldometer: Coronavirus Updates (2020) https://www.world ometers.info/coronavirus/. https://doi.org/10.1101/2020.01.23. 20018549V2

18. John Hopkins University: COVID-19 Maps—John Hopkins Coronavirus Research Center (2020) https://coronavirus.jhu. edu/map.html

19. Fang Y, Zhang H, Xie J, Lin M, Ying L, Pang P, Ji W (2020) Sensitivity of chest CT for COVID-19: comparison to RT-PCR. Radiology. https://doi.org/10.1148/radiol.2020200432

20. Ai T, Yang Z, Hou H, Zhan C, Chen C, Lv W, Tao Q, Sun Z, Xia L (2020) Correlation of chest CT and RT-PCR testing in coronavirus disease 2019 (COVID-19) in China: a report of 1014 cases. Radiology 2019:200642. https://doi.org/10.1148/radiol. 2020200642

21. Shorten C, Khoshgoftaar TM (2019) A survey on image data augmentation for deep learning. J Big Data 6:60. https://doi.org/ 10.1186/s40537-019-0197-0

22. Huang C, Wang Y, Li X, Ren L, Zhao J, Hu Y, Zhang L, Fan G, Xu J, Gu X, Cheng Z, Yu T, Xia J, Wei Y, Wu W, Xie X, Yin W, Li H, Liu M, Xiao Y, Gao H, Guo L, Xie J, Wang G, Jiang R, Gao Z, Jin Q, Wang J, Cao B (2020) Clinical features of patients infected with 2019 novel coronavirus in Wuhan, China. Lancet 395:497-506. https://doi.org/10.1016/S0140-6736(20)30183-5

23. Chung M, Bernheim A, Mei X, Zhang N, Huang M, Zeng X, Cui J, Xu W, Yang Y, Fayad ZA, Jacobi A, Li K, Li S, Shan H (2020) CT imaging features of 2019 novel coronavirus (2019-NCoV). Radiology 295:202-207. https://doi.org/10.1148/radiol.20202 00230

24. Shortliffe EH (1976) Design considerations for MYCIN. In: Computer-Based Medical Consultations: Mycin. Elsevier, pp 63-78. https://doi.org/10.1016/B978-0-444-00179-5.50008-1.

25. Yahyaoui A, Jamil A, Rasheed J, Yesiltepe M (2019) A decision support system for diabetes prediction using machine learning and deep learning techniques. In: 2019 1st international informatics and software engineering conference (UBMYK). IEEE, pp 1-4. https://doi.org/10.1109/UBMYK48245.2019.8965556.

26. Kannadasan K, Edla DR, Kuppili V (2019) Type 2 diabetes data classification using stacked autoencoders in deep neural networks. Clin Epidemiol Glob Health 7:530-535. https://doi.org/ 10.1016/j.cegh.2018.12.004

27. Vaka AR, Soni B, Sudheer Reddy K (2020) Breast cancer detection by leveraging Machine Learning. ICT Express. https://doi. org/10.1016/j.icte.2020.04.009

28. Gárate-Escamila AK, Hajjam El Hassani A, Andrès E (2020) Classification models for heart disease prediction using feature selection and PCA. Inf Med Unlocked 19:100330. https://doi. org/10.1016/j.imu.2020.100330

29. Rasheed J, Hameed AA, Ajlouni N, Jamil A, Ozyavas A, Orman Z (2020) Application of adaptive back-propagation neural networks for Parkinson's disease prediction. In: 2020 International conference on data analytics for business and industry: way towards a sustainable economy (ICDABI). IEEE, pp 1-5. https://doi.org/10.1109/ICDABI51230.2020.9325709.

30. Chockanathan U, DSouza AM, Abidin AZ, Schifitto G, Wismüller A (2019) Automated diagnosis of HIV-associated neurocognitive disorders using large-scale Granger causality analysis of resting-state functional MRI. Comput Biol Med 106:24-30. https://doi.org/10.1016/j.compbiomed.2019.01.006

31. Colubri A, Hartley M-A, Siakor M, Wolfman V, Felix A, Sesay T, Shaffer JG, Garry RF, Grant DS, Levine AC, Sabeti PC (2019) Machine-learning prognostic models from the 201416 Ebola outbreak: data-harmonization challenges, validation strategies, and mHealth applications. EClinicalMedicine 11:54-64. https://doi.org/10.1016/j.eclinm.2019.06.003

32. Gao F, You J, Wang J, Sun J, Yang E, Zhou H (2017) A novel target detection method for SAR images based on shadow proposal and saliency analysis. Neurocomputing 267:220-231. https://doi.org/10.1016/j.neucom.2017.06.004

33. Li H-C, Yang G, Yang W, Du Q, Emery WJ (2020) Deep nonsmooth nonnegative matrix factorization network with semisupervised learning for SAR image change detection. ISPRS J Photogramm Remote Sens 160:167-179. https://doi.org/10. 1016/j.isprsjprs.2019.12.002

34. Pandey MK, Subbiah K (2018) Performance analysis of time series forecasting using Machine Learning algorithms for prediction of Ebola casualties. In: Communications in computer and information science, pp 320-334.https://doi.org/10.1007/ 978-981-13-2035-4_28

35. Pham Q-V, Nguyen DC, Huynh-The T, Hwang W-J, Pathirana PN (2020) Artificial intelligence (AI) and big data for coronavirus (COVID-19) pandemic: a survey on the state-of-thearts. IEEE Access 8:130820-130839. https://doi.org/10.1109/ ACCESS.2020.3009328

36. Rasheed J, Jamil A, Hameed AA, Aftab U, Aftab J, Shah SA, Draheim D (2020) A survey on artificial intelligence approaches in supporting frontline workers and decision makers for the COVID-19 pandemic. Chaos Solitons Fractals 141:110337. https://doi.org/10.1016/j.chaos.2020.110337

37. Bansal A, Padappayil RP, Garg C, Singal A, Gupta M, Klein A (2020) Utility of artificial intelligence amidst the COVID 19 pandemic: a review. J Med Syst 44:156. https://doi.org/10. 1007/s10916-020-01617-3

38. Kumar A, Gupta PK, Srivastava A (2020) A review of modern technologies for tackling COVID-19 pandemic. Diabetes Metab Syndr 14:569-573. https://doi.org/10.1016/j.dsx.2020. 05.008

39. Lalmuanawma S, Hussain J, Chhakchhuak L (2020) Applications of machine learning and artificial intelligence for COVID19 (SARS-CoV-2) pandemic: a review. Chaos Solitons Fractals 139:110059. https://doi.org/10.1016/j.chaos.2020.110059

40. Hussain AA, Bouachir O, Al-Turjman F, Aloqaily M (2020) AI techniques for COVID-19. IEEE Access 8:128776-128795. https://doi.org/10.1109/ACCESS.2020.3007939

41. Swapnarekha H, Behera HS, Nayak J, Naik B (2020) Role of intelligent computing in COVID-19 prognosis: a state-of-the-art review. Chaos Solitons Fractals 138:109947. https://doi.org/10. 1016/j.chaos.2020.109947

42. Waleed Salehi A, Baglat P, Gupta G (2020) Review on machine and deep learning models for the detection and prediction of Coronavirus. Mater Today Proc. https://doi.org/10.1016/j.matpr. 2020.06.245 
43. Jamshidi MB, Lalbakhsh A, Talla J, Peroutka Z, Hadjilooei F, Lalbakhsh P, Jamshidi M, Spada LL, Mirmozafari M, Dehghani M, Sabet A, Roshani S, Roshani S, Bayat-Makou N, Mohamadzade B, Malek Z, Jamshidi A, Kiani S, Hashemi-Dezaki H, Mohyuddin W (2020) Artificial intelligence and COVID-19: deep learning approaches for diagnosis and treatment. IEEE Access 8:109581-109595. https://doi.org/10.1109/ACCESS. 2020.3001973

44. Shinde GR, Kalamkar AB, Mahalle PN, Dey N, Chaki J, Hassanien AE (2020) Forecasting models for coronavirus disease (COVID-19): a survey of the state-of-the-art. SN Comput Sci 1:197. https://doi.org/10.1007/s42979-020-00209-9

45. Albahri AS, Hamid RA, Alwan JK, Al-qays ZT, Zaidan AA, Zaidan BB, Albahri AOS, AlAmoodi AH, Khlaf JM, Almahdi EM, Thabet E, Hadi SM, Mohammed KI, Alsalem MA, AlObaidi JR, Madhloom HT (2020) Role of biological data mining and machine learning techniques in detecting and diagnosing the novel coronavirus (COVID-19): a systematic review. J Med Syst 44:122. https://doi.org/10.1007/s10916-020-01582-x

46. Ahmad A, Garhwal S, Ray SK, Kumar G, Malebary SJ, Barukab OM (2020) The number of confirmed cases of COVID-19 by using machine learning: methods and challenges. Arch Comput Methods Eng. https://doi.org/10.1007/s11831-020-09472-8

47. Monshi MMA, Poon J, Chung V (2020) Deep learning in generating radiology reports: a survey. Artif Intell Med 106:101878. https://doi.org/10.1016/j.artmed.2020.101878

48. Jalaber C, Lapotre T, Morcet-Delattre T, Ribet F, Jouneau S, Lederlin M (2020) Chest CT in COVID-19 pneumonia: a review of current knowledge. Diagn Interv Imaging 101:431-437. https:// doi.org/10.1016/j.diii.2020.06.001

49. Shaikh F, Andersen MB, Sohail MR, Mulero F, Awan O, DupontRoettger D, Kubassova O, Dehmeshki J, Bisdas S (2020) Current landscape of imaging and the potential role for artificial intelligence in the management of COVID-19. Curr Probl Diagn Radiol. https://doi.org/10.1067/j.cpradiol.2020.06.009

50. Dong D, Tang Z, Wang S, Hui H, Gong L, Lu Y, Xue Z, Liao H, Chen F, Yang F, Jin R, Wang K, Liu Z, Wei J, Mu W, Zhang H, Jiang J, Tian J, Li H (2020) The role of imaging in the detection and management of COVID-19: a review. IEEE Rev Biomed Eng. https://doi.org/10.1109/RBME.2020.2990959

51. Shi F, Wang J, Shi J, Wu Z, Wang Q, Tang Z, He K, Shi Y, Shen D (2020) Review of artificial intelligence techniques in imaging data acquisition, segmentation and diagnosis for COVID19. IEEE Rev Biomed Eng. https://doi.org/10.1109/RBME.2020. 2987975

52. Cui F, Zhou HS (2020) Diagnostic methods and potential portable biosensors for coronavirus disease 2019. Biosens Bioelectron 165:112349. https://doi.org/10.1016/j.bios.2020.112349

53. Swayamsiddha S, Mohanty C (2020) Application of cognitive Internet of Medical Things for COVID-19 pandemic. Diabetes Metab Syndr 14:911-915. https://doi.org/10.1016/j.dsx.2020.06. 014

54. Panch T, Szolovits P, Atun R (2018) Artificial intelligence, machine learning and health systems. J Glob Health 8:1-8. https://doi.org/10.7189/jogh.08.020303

55. Sethy PK, Behera SK, Ratha PK, Biswas P (2020) Detection of coronavirus disease (COVID-19) based on deep features and support vector machine. Int J Math Eng Manage Sci 5:643-651. https://doi.org/10.33889/IJMEMS.2020.5.4.052

56. Öztürk Ş, Özkaya U, Barstuğan M (2020) Classification of Coronavirus (COVID-19) from X-ray and CT images using shrunken features. Int J Imaging Syst Technol. https://doi.org/10.1002/ima. 22469

57. Kang H, Xia L, Yan F, Wan Z, Shi F, Yuan H, Jiang H, Wu D, Sui H, Zhang C, Shen D (2020) Diagnosis of coronavirus disease 2019 (COVID-19) with structured latent multi-view representation learning. IEEE Trans Med Imaging 39:26062614. https://doi.org/10.1109/TMI.2020.2992546

58. Milletari F, Navab N, Ahmadi S-A (2016) V-Net: fully convolutional neural networks for volumetric medical image segmentation. In: 2016 fourth international conference on 3D vision (3DV). IEEE, pp 565-571. https://doi.org/10.1109/3DV.2016. 79.

59. Zhang C, Han Z, Cui Y, Fu H, Zhou JT, Hu Q (2019) CPM-nets: cross partial multi-view networks. NeurIPS 559-569

60. Sun L, Mo Z, Yan F, Xia L, Shan F, Ding Z, Song B, Gao W, Shao W, Shi F, Yuan H, Jiang H, Wu D, Wei Y, Gao Y, Sui H, Zhang D, Shen D (2020) Adaptive feature selection guided deep forest for COVID-19 classification with chest CT. IEEE J Biomed Health Inf. https://doi.org/10.1109/JBHI.2020.3019505

61. Bahadur Chandra T, Verma K, Kumar Singh B, Jain D, Singh Netam S (2020) Coronavirus disease (COVID-19) detection in chest X-ray images using majority voting based classifier ensemble. Exp Syst Appl. https://doi.org/10.1016/j.eswa.2020.113909

62. Yoo SH, Geng H, Chiu TL, Yu SK, Cho DC, Heo J, Choi MS, Choi IH, Van Cung C, Nhung NV, Min BJ, Lee H (2020) Deep learning-based decision-tree classifier for COVID-19 diagnosis from chest X-ray imaging. Front Med. https://doi.org/10.3389/ fmed.2020.00427

63. Toğaçar M, Ergen B, Cömert Z (2020) COVID-19 detection using deep learning models to exploit Social Mimic Optimization and structured chest X-ray images using fuzzy color and stacking approaches. Comput Biol Med. https://doi.org/10.1016/j.compb iomed.2020.103805

64. Pereira RM, Bertolini D, Teixeira LO, Silla CN, Costa YMG (2020) COVID-19 identification in chest X-ray images on flat and hierarchical classification scenarios. Comput Methods Programs Biomed 194:105532. https://doi.org/10.1016/j.cmpb.2020. 105532

65. Tuncer T, Dogan S, Ozyurt F (2020) An automated residual exemplar local binary pattern and iterative relief based corona detection method using lung X-ray image. Chemom Intell Lab Syst 203:104054. https://doi.org/10.1016/j.chemolab.2020. 104054

66. Abbasian Ardakani A, Acharya UR, Habibollahi S, Mohammadi A (2020) COVIDiag: a clinical CAD system to diagnose COVID19 pneumonia based on CT findings. Eur Radiol. https://doi.org/ 10.1007/s00330-020-07087-y

67. Apostolopoulos ID, Mpesiana TA (2020) Covid-19: automatic detection from X-ray images utilizing transfer learning with convolutional neural networks. Phys Eng Sci Med 43:635-640. https://doi.org/10.1007/s13246-020-00865-4

68. Brunese L, Mercaldo F, Reginelli A, Santone A (2020) Explainable deep learning for pulmonary disease and coronavirus COVID-19 detection from X-rays. Comput Methods Programs Biomed 196:105608. https://doi.org/10.1016/j.cmpb.2020. 105608

69. Rasheed J, Hameed AA, Djeddi C, Jamil A, Al-Turjman F (2021) A machine learning-based framework for diagnosis of COVID19 from chest X-ray images. Interdiscip Sci Comput Life Sci. https://doi.org/10.1007/s12539-020-00403-6

70. Xu X, Jiang X, Ma C, Du P, Li X, Lv S, Yu L, Ni Q, Chen Y, Su J, Lang G, Li Y, Zhao H, Liu J, Xu K, Ruan L, Sheng J, Qiu Y, Wu W, Liang T, Li L (2020) A Deep learning system to screen novel coronavirus disease 2019 pneumonia. Engineering. https:// doi.org/10.1016/j.eng.2020.04.010

71. Jaiswal A, Gianchandani N, Singh D, Kumar V, Kaur M (2020) Classification of the COVID-19 infected patients using DenseNet201 based deep transfer learning. J Biomol Struct Dyn. https://doi.org/10.1080/07391102.2020.1788642

72. Ahuja S, Panigrahi BK, Dey N, Rajinikanth V, Gandhi TK (2020) Deep transfer learning-based automated detection of COVID-19 
from lung CT scan slices. Appl Intell. https://doi.org/10.1007/ s10489-020-01826-w

73. Perumal V, Narayanan V, Rajasekar SJS (2020) Detection of COVID-19 using CXR and CT images using Transfer Learning and Haralick features. Appl Intell. https://doi.org/10.1007/ s10489-020-01831-z

74. Bridge J, Meng Y, Zhao Y, Du Y, Zhao M, Sun R, Zheng Y (2020) Introducing the GEV activation function for highly unbalanced data to develop COVID-19 diagnostic models. IEEE J Biomed Health Inf. https://doi.org/10.1109/JBHI.2020.3012383

75. Fan D-P, Zhou T, Ji G-P, Zhou Y, Chen G, Fu H, Shen J, Shao L (2020) Inf-net: automatic COVID-19 lung infection segmentation from CT images. IEEE Trans Med Imaging 39:2626-2637. https://doi.org/10.1109/TMI.2020.2996645

76. Wang J, Bao Y, Wen Y, Lu H, Luo H, Xiang Y, Li X, Liu C, Qian D (2020) Prior-attention residual learning for more discriminative COVID-19 screening in CT images. IEEE Trans Med Imaging 39:2572-2583. https://doi.org/10.1109/TMI.2020.2994908

77. Mohammed A, Wang C, Zhao M, Ullah M, Naseem R, Wang H, Pedersen M, Cheikh FA (2020) Semi-supervised network for detection of COVID-19 in chest CT scans. IEEE Access. https:// doi.org/10.1109/ACCESS.2020.3018498

78. Ouyang X, Huo J, Xia L, Shan F, Liu J, Mo Z, Yan F, Ding Z, Yang Q, Song B, Shi F, Yuan H, Wei Y, Cao X, Gao Y, Wu D, Wang Q, Shen D (2020) Dual-sampling attention network for diagnosis of COVID-19 from community acquired pneumonia. IEEE Trans Med Imaging 39:2595-2605. https://doi.org/10. 1109/TMI.2020.2995508

79. Han Z, Wei B, Hong Y, Li T, Cong J, Zhu X, Wei H, Zhang W (2020) Accurate screening of COVID-19 using attention-based deep 3D multiple instance learning. IEEE Trans Med Imaging 39:2584-2594. https://doi.org/10.1109/TMI.2020.2996256

80. Harmon SA, Sanford TH, Xu S, Turkbey EB, Roth H, Xu Z, Yang D, Myronenko A, Anderson V, Amalou A, Blain M, Kassin M, Long D, Varble N, Walker SM, Bagci U, Ierardi AM, Stellato E, Plensich GG, Franceschelli G, Girlando C, Irmici G, Labella D, Hammoud D, Malayeri A, Jones E, Summers RM, Choyke PL, Xu D, Flores M, Tamura K, Obinata H, Mori H, Patella F, Cariati M, Carrafiello G, An P, Wood BJ, Turkbey B (2020) Artificial intelligence for the detection of COVID-19 pneumonia on chest CT using multinational datasets. Nat Commun 11:4080. https:// doi.org/10.1038/s41467-020-17971-2

81. Sharma S (2020) Drawing insights from COVID-19-infected patients using CT scan images and machine learning techniques: a study on 200 patients. Environ Sci Pollut Res. https://doi.org/ 10.1007/s11356-020-10133-3

82. Yan T, Wong PK, Ren H, Wang H, Wang J, Li Y (2020) Automatic distinction between COVID-19 and common pneumonia using multi-scale convolutional neural network on chest CT scans. Chaos Solitons Fractals 140:110153. https://doi.org/10. 1016/j.chaos.2020.110153

83. Dey N, Rajinikanth V, Fong SJ, Kaiser MS, Mahmud M (2020) Social group optimization-assisted Kapur's entropy and morphological segmentation for automated detection of COVID-19 infection from computed tomography images. Cogn Comput. https://doi.org/10.1007/s12559-020-09751-3

84. Wang S, Zha Y, Li W, Wu Q, Li X, Niu M, Wang M, Qiu X, Li H, Yu H, Gong W, Bai Y, Li L, Zhu Y, Wang L, Tian J (2020) A fully automatic deep learning system for COVID-19 diagnostic and prognostic analysis. Eur Respir J. https://doi.org/10.1183/ 13993003.00775-2020

85. Ni Q, Sun ZY, Qi L, Chen W, Yang Y, Wang L, Zhang X, Yang L, Fang Y, Xing Z, Zhou Z, Yu Y, Lu GM, Zhang LJ (2020) A deep learning approach to characterize 2019 coronavirus disease (COVID-19) pneumonia in chest CT images. Eur Radiol. https:// doi.org/10.1007/s00330-020-07044-9
86. Pu J, Leader J, Bandos A, Shi J, Du P, Yu J, Yang B, Ke S, Guo Y, Field JB, Fuhrman C, Wilson D, Sciurba F, Jin C (2020) Any unique image biomarkers associated with COVID-19? Eur Radiol. https://doi.org/10.1007/s00330-020-06956-w

87. Mei X, Lee HC, Diao KY, Huang M, Lin B, Liu C, Xie Z, Ma Y, Robson PM, Chung M, Bernheim A, Mani V, Calcagno C, Li K, Li S, Shan H, Lv J, Zhao T, Xia J, Long Q, Steinberger S, Jacobi A, Deyer T, Luksza M, Liu F, Little BP, Fayad ZA, Yang Y (2020) Artificial intelligence-enabled rapid diagnosis of patients with COVID-19. Nat Med. https://doi.org/10.1038/ s41591-020-0931-3

88. Pathak Y, Shukla PK, Tiwari A, Stalin S, Singh S (2020) Deep transfer learning based classification model for COVID-19 disease. Irbm 1:1-6. https://doi.org/10.1016/j.irbm.2020.05.003

89. Zhang K, Liu X, Shen J, Li Z, Sang Y, Wu X, Zha Y, Liang W, Wang C, Wang K, Ye L, Gao M, Zhou Z, Li L, Wang J, Yang Z, Cai H, Xu J, Yang L, Cai W, Xu W, Wu S, Zhang W, Jiang S, Zheng L, Zhang X, Wang L, Lu L, Li J, Yin H, Wang W, Li O, Zhang C, Liang L, Wu T, Deng R, Wei K, Zhou Y, Chen T, Lau JYN, Fok M, He J, Lin T, Li W, Wang G (2020) Clinically applicable AI system for accurate diagnosis, quantitative measurements, and prognosis of COVID-19 pneumonia using computed tomography. Cell 181:1423-1433.e11. https://doi.org/ 10.1016/j.cell.2020.04.045

90. Wu X, Hui H, Niu M, Li L, Wang L, He B, Yang X, Li L, Li H, Tian J, Zha Y (2020) Deep learning-based multi-view fusion model for screening 2019 novel coronavirus pneumonia: a multicentre study. Eur J Radiol 128:109041. https://doi.org/10.1016/j. ejrad.2020.109041

91. Bai HX, Wang R, Xiong Z, Hsieh B, Chang K, Halsey K, Tran TML, Choi JW, Wang D-C, Shi L-B, Mei J, Jiang X-L, Pan I, Zeng Q-H, Hu P-F, Li Y-H, Fu F-X, Huang RY, Sebro R, Yu Q-Z, Atalay MK, Liao W-H (2020) AI augmentation of radiologist performance in distinguishing COVID-19 from pneumonia of other etiology on chest CT. Radiology 78:201491. https://doi. org/10.1148/radiol.2020201491

92. Butt C, Gill J, Chun D, Babu BA (2020) Deep learning system to screen coronavirus disease 2019 pneumonia. Appl Intell. https:// doi.org/10.1007/s10489-020-01714-3

93. Akif Cifci M (2020) Deep learning model for diagnosis of corona virus disease from CT images. Int J Sci Eng Res 11:273-278

94. Singh D, Kumar V, Vaishali, Kaur M (2020) Classification of COVID-19 patients from chest CT images using multi-objective differential evolution-based convolutional neural networks. Eur J Clin Microbiol Infect Dis. https://doi.org/10.1007/ s10096-020-03901-Z

95. Farid AA, Selim GI, Awad H, Khater A (2020) A novel approach of CT images feature analysis and prediction to screen for corona virus disease (COVID-19). Int J Sci Eng Res. https://doi.org/10. 14299/ijser.2020.03.02

96. Li L, Qin L, Xu Z, Yin Y, Wang X, Kong B, Bai J, Lu Y, Fang Z, Song Q, Cao K, Liu D, Wang G, Xu Q, Fang X, Zhang S, Xia J, Xia J (2020) Using artificial intelligence to detect COVID-19 and community-acquired pneumonia based on pulmonary CT: evaluation of the diagnostic accuracy. Radiology 296:E65-E71. https://doi.org/10.1148/radiol.2020200905

97. Toraman S, Alakus TB, Turkoglu I (2020) Convolutional capsnet: a novel artificial neural network approach to detect COVID-19 disease from X-ray images using capsule networks. Chaos Solitons Fractals 140:110122. https://doi.org/10.1016/j.chaos.2020. 110122

98. Altan A, Karasu S (2020) Recognition of COVID-19 disease from X-ray images by hybrid model consisting of 2D curvelet transform, chaotic salp swarm algorithm and deep learning technique. Chaos, Solitons Fractals. https://doi.org/10.1016/j.chaos. 2020.110071 
99. Narayan Das N, Kumar N, Kaur M, Kumar V, Singh D (2020) Automated deep transfer learning-based approach for detection of COVID-19 infection in chest X-rays. Irbm 1:1-6. https://doi. org/10.1016/j.irbm.2020.07.001

100. Chowdhury MEH, Rahman T, Khandakar A, Mazhar R, Kadir MA, Mahbub ZB, Islam KR, Khan MS, Iqbal A, Al Emadi N, Reaz MBI, Islam MT (2020) Can AI help in screening viral and COVID-19 pneumonia? IEEE Access 8:132665-132676. https:// doi.org/10.1109/ACCESS.2020.3010287

101. Shibly KH, Dey SK, Islam MT-U, Rahman MM (2020) COVID faster R-CNN: a novel framework to diagnose novel coronavirus disease (COVID-19) in X-ray images. Inform Med Unlocked 20:100405. https://doi.org/10.1016/j.imu.2020.100405

102. Ko H, Chung H, Kang WS, Kim KW, Shin Y, Kang SJ, Lee JH, Kim YJ, Kim NY, Jung H, Lee J (2020) COVID-19 pneumonia diagnosis using a simple 2D deep learning framework with a single chest CT image: model development and validation. J Med Internet Res 22:e19569. https://doi.org/10.2196/19569

103. Das D, Santosh KC, Pal U (2020) Truncated inception net: COVID-19 outbreak screening using chest X-rays. Phys Eng Sci Med. https://doi.org/10.1007/s13246-020-00888-x

104. Bharati S, Podder P, Mondal MRH (2020) Hybrid deep learning for detecting lung diseases from X-ray images. Inform Med Unlocked 20:100391. https://doi.org/10.1016/j.imu.2020.100391

105. Pandit MK, Banday SA (2020) SARS n-CoV2-19 detection from chest $\mathrm{X}$-ray images using deep neural networks. Int J Pervasive Comput Commun. https://doi.org/10.1108/IJPCC-06-2020-0060

106. Khan AI, Shah JL, Bhat MM (2020) CoroNet: a deep neural network for detection and diagnosis of COVID-19 from chest x-ray images. Comput Methods Programs Biomed 196:105581. https://doi.org/10.1016/j.cmpb.2020.105581

107. Mahmud T, Rahman MA, Fattah SA (2020) CovXNet: a multidilation convolutional neural network for automatic COVID-19 and other pneumonia detection from chest X-ray images with transferable multi-receptive feature optimization. Comput Biol Med 122:103869. https://doi.org/10.1016/j.compbiomed.2020. 103869

108. Rajaraman S, Siegelman J, Alderson PO, Folio LS, Folio LR, Antani SK (2020) Iteratively pruned deep learning ensembles for COVID-19 detection in chest X-rays. IEEE Access. https:// doi.org/10.1109/ACCESS.2020.3003810

109. Waheed A, Goyal M, Gupta D, Khanna A, Al-Turjman F, Pinheiro PR (2020) CovidGAN: data augmentation using auxiliary classifier GAN for improved COVID-19 detection. IEEE Access 8:91916-91923. https://doi.org/10.1109/ACCESS.2020.2994762

110. Apostolopoulos ID, Aznaouridis SI, Tzani MA (2020) Extracting possibly representative COVID-19 biomarkers from X-ray images with deep learning approach and image data related to pulmonary diseases. J Med Biol Eng 40:462-469. https://doi.org/ 10.1007/s40846-020-00529-4

111. Rahimzadeh M, Attar A (2020) A modified deep convolutional neural network for detecting COVID-19 and pneumonia from chest X-ray images based on the concatenation of Xception and ResNet50V2. Inform Med Unlocked 19:100360. https://doi.org/ 10.1016/j.imu.2020.100360

112. Rajaraman S, Antani S (2020) Weakly labeled data augmentation for deep learning: a study on COVID-19 detection in chest X-rays. Diagnostics 10:358. https://doi.org/10.3390/diagnostic s10060358

113. Tsiknakis N, Trivizakis E, Vassalou E, Papadakis G, Spandidos D, Tsatsakis A, Sánchez-García J, López-González R, Papanikolaou N, Karantanas A, Marias K (2020) Interpretable artificial intelligence framework for COVID-19 screening on chest X-rays. Exp Ther Med. https://doi.org/10.3892/etm.2020.8797

114. Loey M, Smarandache F, Khalifa NEM (2020) Within the lack of chest COVID-19 X-ray dataset: a novel detection model based on GAN and deep transfer learning. Symmetry. https://doi.org/ 10.3390/SYM12040651

115. Ozturk T, Talo M, Yildirim EA, Baloglu UB, Yildirim O, Rajendra Acharya U (2020) Automated detection of COVID-19 cases using deep neural networks with X-ray images. Comput Biol Med 121:103792. https://doi.org/10.1016/j.compbiomed.2020. 103792

116. Ucar F, Korkmaz D (2020) COVIDiagnosis-Net: Deep BayesSqueezeNet based diagnosis of the coronavirus disease 2019 (COVID-19) from X-ray images. Med Hypotheses 140:109761. https://doi.org/10.1016/j.mehy.2020.109761

117. Sedik A, Iliyasu AM, Abd El-Rahiem B, Abdel Samea ME, Abdel-Raheem A, Hammad M, Peng J, Abd El-Samie FE, Abd El-Latif AA (2020) Deploying machine and deep learning models for efficient data-augmented detection of COVID-19 infections. Viruses 12:769. https://doi.org/10.3390/v12070769

118. Hwang EJ, Kim H, Yoon SH, Goo JM, Park CM (2020) Implementation of a deep learning-based computer-aided detection system for the interpretation of chest radiographys in patients suspected for COVID-19. Korean J Radiol 21:1-11. https://doi. org/10.3348/kjr.2020.0536

119. de Moraes Batista AF, Miraglia JL, Donato THR, Filho ADPC (2020) COVID-19 diagnosis prediction in emergency care patients: a machine learning approach. medRxiv. https://doi.org/ 10.1101/2020.04.04.20052092

120. Brinati D, Campagner A, Ferrari D, Locatelli M, Banfi G, Cabitza F (2020) Detection of COVID-19 infection from routine blood exams with machine learning: a feasibility study. J Med Syst 44:135. https://doi.org/10.1007/s10916-020-01597-4

121. Yang HS, Vasovic LV, Steel P, Chadburn A, Hou Y, RacineBrzostek SE, Cushing M, Loda M, Kaushal R, Zhao Z, Wang F (2020) Routine laboratory blood tests predict SARS-CoV-2 infection using machine learning. medRxiv. https://doi.org/10. 1101/2020.06.17.20133892

122. Khanday AMUD, Rabani ST, Khan QR, Rouf N, Mohiuddin M (2020) Machine learning based approaches for detecting COVID19 using clinical text data. Int J Inf Technol. https://doi.org/10. 1007/s41870-020-00495-9

123. Wu J, Zhang P, Zhang L, Meng W, Li J, Tong C, Li Y, Cai J, Yang Z, Zhu J, Zhao M, Huang H, Xie X, Li S (2020) Rapid and accurate identification of COVID-19 infection through machine learning based on clinical available blood test results. medRxiv. https://doi.org/10.1101/2020.04.02.20051136

124. Wang Y, Hu M, Li Q, Zhang X-P, Zhai G, Yao N (2020) Abnormal respiratory patterns classifier may contribute to large-scale screening of people infected with COVID-19 in an accurate and unobtrusive manner

125. Cho K, van Merrienboer B, Gulcehre C, Bahdanau D, Bougares F, Schwenk H, Bengio Y (2014) Learning phrase representations using RNN encoder-decoder for statistical machine translation. In: Proceedings of the 2014 conference on empirical methods in natural language processing (EMNLP). Association for Computational Linguistics, Stroudsburg, pp 1724-1734. https://doi.org/ 10.3115/v1/D14-1179

126. Imran A, Posokhova I, Qureshi HN, Masood U, Riaz S, Ali K, John CN, Hussain I, Nabeel M (2020) AI4COVID-19: AI enabled preliminary diagnosis for COVID-19 from cough samples via an app. Inform Med Unlocked. https://doi.org/10.1016/j.imu. 2020.100378

127. Brown C, Chauhan J, Grammenos A, Han J, Hasthanasombat A, Spathis D, Xia T, Cicuta P, Mascolo C (2020) Exploring automatic diagnosis of COVID-19 from crowdsourced respiratory sound data. In: 26th SIGKDD conference on knowledge discovery and data mining. https://doi.org/10.17863/CAM.55747.

128. Jiang Z, Hu M, Gao Z, Fan L, Dai R, Pan Y, Tang W, Zhai G, Lu $Y$ (2020) Detection of respiratory infections using RGB-infrared 
sensors on portable device. IEEE Sensors J. https://doi.org/10. 1109/JSEN.2020.3004568

129. Carrer L, Donini E, Marinelli D, Zanetti M, Mento F, Torri E, Smargiassi A, Inchingolo R, Soldati G, Demi L, Bovolo F, Bruzzone L (2020) Automatic pleural line extraction and COVID-19 scoring from lung ultrasound data. IEEE Trans Ultrason Ferroelect Freq Contr. https://doi.org/10.1109/TUFFC.2020.3005512

130. Zhou L, Li Z, Zhou J, Li H, Chen Y, Huang Y, Xie D, Zhao L, Fan M, Hashmi S, Abdelkareem F, Eiada R, Xiao X, Li L, Qiu Z, Gao X (2020) A rapid, accurate and machine-agnostic segmentation and quantification method for CT-based COVID-19 diagnosis. IEEE Trans Med Imaging 39:2638-2652. https://doi. org/10.1109/TMI.2020.3001810

131. Bai X, Fang C, Zhou Y, Bai S, Liu Z, Xia L, Chen Q, Xu Y, Xia T, Gong S, Xie X, Song D, Du R, Zhou C, Chen C, Nie D, Qin L, Chen W (2020) Predicting COVID-19 malignant progression with AI techniques. SSRN Electron J. https://doi.org/10.2139/ ssrn.3557984

132. Albahri OS, Al-Obaidi JR, Zaidan AA, Albahri AS, Zaidan BB, Salih MM, Qays A, Dawood KA, Mohammed RT, Abdulkareem KH, Aleesa AM, Alamoodi AH, Chyad MA, Zulkifli CZ (2020) Helping doctors hasten COVID-19 treatment: towards a rescue framework for the transfusion of best convalescent plasma to the most critical patients based on biological requirements via $\mathrm{ml}$ and novel MCDM methods. Comput Methods Programs Biomed 196:105617. https://doi.org/10.1016/j.cmpb.2020.105617

133. Lessmann N, Sánchez CI, Beenen L, Boulogne LH, Brink M, Calli E, Charbonnier J-P, Dofferhoff T, van Everdingen WM, Gerke PK, Geurts B, Gietema HA, Groeneveld M, van Harten L, Hendrix N, Hendrix W, Huisman HJ, Išgum I, Jacobs C, Kluge R, Kok M, Krdzalic J, Lassen-Schmidt B, van Leeuwen K, Meakin J, Overkamp M, van Rees Vellinga T, van Rikxoort EM, Samperna R, Schaefer-Prokop C, Schalekamp S, Scholten ET, Sital C, Stöger L, Teuwen J, Vaidhya Venkadesh K, de Vente C, Vermaat M, Xie W, de Wilde B, Prokop M, van Ginneken B (2020) Automated assessment of CO-RADS and chest CT severity scores in patients with suspected COVID-19 using artificial intelligence. Radiology. https://doi.org/10.1148/radiol.20202 02439

134. Zhang H, Zhang J, Zhang H, Nan Y, Zhao Y, Fu E, Xie Y, Liu W, Li W, Zhang H, Jiang H, Li C, Li Y, Ma R, Dang S, Gao B, Zhang X, Zhang T (2020) Automated detection and quantification of COVID-19 pneumonia: CT imaging analysis by a deep learning-based software. Eur J Nucl Med Mol Imaging. https:// doi.org/10.1007/s00259-020-04953-1

135. Zhu JS, Ge P, Jiang C, Zhang Y, Li X, Zhao Z, Zhang L, Duong TQ (2020) Deep-learning artificial intelligence analysis of clinical variables predicts mortality in COVID-19 patients. J Am Coll Emerg Phys Open. https://doi.org/10.1002/emp2.12205

136. Zhu J, Shen B, Abbasi A, Hoshmand-Kochi M, Li H, Duong TQ (2020) Deep transfer learning artificial intelligence accurately stages COVID-19 lung disease severity on portable chest radiographs. PLoS ONE 15:e0236621. https://doi.org/10.1371/journ al.pone. 0236621

137. Malki Z, Atlam ES, Hassanien AE, Dagnew G, Elhosseini MA, Gad I (2020) Association between weather data and COVID19 pandemic predicting mortality rate: machine learning approaches. Chaos Solitons Fractals 138:110137. https://doi. org/10.1016/j.chaos.2020.110137

138. Hassantabar S, Ahmadi M, Sharifi A (2020) Diagnosis and detection of infected tissue of COVID-19 patients based on lung X-ray image using convolutional neural network approaches. Chaos Solitons Fractals 140:110170. https://doi.org/10.1016/j.chaos. 2020.110170

139. Cohen JP, Dao L, Roth K, Morrison P, Bengio Y, Abbasi AF, Shen B, Mahsa HK, Ghassemi M, Li H, Duong T (2020)
Predicting COVID-19 pneumonia severity on chest X-ray with deep learning. Cureus. https://doi.org/10.7759/cureus.9448

140. Iwendi C, Bashir AK, Peshkar A, Chatterjee JM, Pasupuleti S, Mishra R, Pillai S, Jo O (2020) COVID-19 patient health prediction using boosted random forest algorithm. Front Public Health 8:1-9. https://doi.org/10.3389/fpubh.2020.00357

141. Matos J, Paparo F, Mussetto I, Bacigalupo L, Veneziano A, Perugin Bernardi S, Biscaldi E, Melani E, Antonucci G, Cremonesi P, Lattuada M, Pilotto A, Pontali E, Rollandi GA (2020) Evaluation of novel coronavirus disease (COVID-19) using quantitative lung $\mathrm{CT}$ and clinical data: prediction of short-term outcome. Eur Radiol Exp. https://doi.org/10.1186/ s41747-020-00167-0

142. Hassanien AE, Salam A, Darwish A, Hassanien AE, Salama A, Darwsih A (2020) Artificial intelligence approach to predict the COVID-19 patient's recovery artificial intelligence approach to predict the COVID-19 patient's recovery. EasyChair 3223

143. Zhang N, Zhang R, Yao H, Xu H, Duan M, Xie T, Pan J, Peng E, Huang J, Zhang Y, Xu X, Zhou F, Wang G (2020) Severity detection for the coronavirus disease 2019 (COVID-19) patients using a machine learning model based on the blood and urine tests. SSRN Electron J. https://doi.org/10.2139/ssrn.3564426

144. Carrillo-Larco RM, Castillo-Cara M (2020) Using countrylevel variables to classify countries according to the number of confirmed COVID-19 cases: an unsupervised machine learning approach. Wellcome Open Res 5:56. https://doi.org/10.12688/ wellcomeopenres. 15819.2

145. Kavadi MDP, Patan DR, Ramachandran DM, Gandomi AH (2020) Partial derivative nonlinear global pandemic machine learning prediction of COVID 19. Chaos Solitons Fractals. https://doi.org/10.1016/j.chaos.2020.110056

146. Hu Z, Ge Q, Li S, Boerwinkle E, Jin L, Xiong M (2020) Forecasting and evaluating multiple interventions for COVID-19 worldwide. Front Artif Intell 3:1-11. https://doi.org/10.3389/ frai.2020.00041

147. Paul SK, Jana S, Bhaumik P (2020) A multivariate spatiotemporal spread model of COVID-19 using ensemble of ConvLSTM networks. medRxiv. https://doi.org/10.1101/2020.04.17.20069 898

148. Kasilingam D, Prabhakaran SS, Dinesh Kumar R, Rajagopal V, Santhosh Kumar T, Soundararaj A (2020) Exploring the growth of COVID-19 cases using exponential modelling across 42 countries and predicting signs of early containment using machine learning. Transbound Emerg Dis. https://doi.org/10.1111/tbed. 13764

149. Shahid F, Zameer A, Muneeb M (2020) Predictions for COVID19 with deep learning models of LSTM GRU and Bi-LSTM. Chaos Solitons Fractals 140:110212. https://doi.org/10.1016/j. chaos.2020.110212

150. Singh S, Parmar KS, Makkhan SJS, Kaur J, Peshoria S, Kumar J (2020) Study of ARIMA and least square support vector machine (LS-SVM) models for the prediction of SARS-CoV-2 confirmed cases in the most affected countries. Chaos Solitons Fractals. https://doi.org/10.1016/j.chaos.2020.110086

151. Zeroual A, Harrou F, Dairi A, Sun Y (2020) Deep learning methods for forecasting COVID-19 time-series data: a comparative study. Chaos Solitons Fractals. https://doi.org/10.1016/j.chaos. 2020.110121

152. Wang P, Zheng X, Li J, Zhu B (2020) Prediction of epidemic trends in COVID-19 with logistic model and machine learning technics. Chaos Solitons Fractals 139:110058. https://doi.org/10. 1016/j.chaos.2020.110058

153. Yadav M, Perumal M, Srinivas M (2020) Analysis on novel coronavirus (COVID-19) using machine learning methods. Chaos Solitons Fractals 139:110050. https://doi.org/10.1016/j.chaos. 2020.110050 
154. Sujath R, Chatterjee JM, Hassanien AE (2020) A machine learning forecasting model for COVID-19 pandemic in India. Stoch Env Res Risk Assess 34:959-972. https://doi.org/10.1007/ s00477-020-01827-8

155. Rustam F, Reshi AA, Mehmood A, Ullah S, On B-W, Aslam W, Choi GS (2020) COVID-19 future forecasting using supervised machine learning models. IEEE Access 8:101489-101499. https://doi.org/10.1109/ACCESS.2020.2997311

156. Melin P, Monica JC, Sanchez D, Castillo O (2020) Analysis of spatial spread relationships of coronavirus (COVID-19) pandemic in the world using self organizing maps. Chaos Solitons Fractals 138:109917. https://doi.org/10.1016/j.chaos.2020. 109917

157. Tuli S, Tuli S, Tuli R, Gill SS (2020) Predicting the growth and trend of COVID-19 pandemic using machine learning and cloud computing. Internet Things 11:100222. https://doi.org/10.1016/j. iot. 2020.100222

158. Ayyoubzadeh SM, Ayyoubzadeh SM, Zahedi H, Ahmadi M, Niakan Kalhori SR (2020) Predicting COVID-19 incidence through analysis of google trends data in Iran: data mining and deep learning pilot study. JMIR Public Health Surveill 6:18828. https://doi.org/10.2196/18828

159. Chakraborty T, Ghosh I (2020) Real-time forecasts and risk assessment of novel coronavirus (COVID-19) cases: a datadriven analysis. Chaos, Solitons Fractals. https://doi.org/10. 1016/j.chaos.2020.109850

160. Fong SJ, Li G, Dey N, Crespo RG, Herrera-Viedma E (2020) Composite Monte Carlo decision making under high uncertainty of novel coronavirus epidemic using hybridized deep learning and fuzzy rule induction. Appl Soft Comput J 93:106282. https:// doi.org/10.1016/j.asoc.2020.106282

161. Tomar A, Gupta N (2020) Prediction for the spread of COVID19 in India and effectiveness of preventive measures. Sci Total Environ 728:138762. https://doi.org/10.1016/j.scitotenv.2020. 138762

162. Fang Y, Nie Y, Penny M (2020) Transmission dynamics of the COVID-19 outbreak and effectiveness of government interventions: a data-driven analysis. J Med Virol 92:645-659. https:// doi.org/10.1002/jmv.25750

163. Yang Z, Zeng Z, Wang K, Wong SS, Liang W, Zanin M, Liu P, Cao X, Gao Z, Mai Z, Liang J, Liu X, Li S, Li Y, Ye F, Guan W,
Yang Y, Li F, Luo S, Xie Y, Liu B, Wang Z, Zhang S, Wang Y, Zhong N, He J (2020) Modified SEIR and AI prediction of the epidemics trend of COVID-19 in China under public health interventions. J Thorac Dis 12:165-174. https://doi.org/10.21037/jtd. 2020.02.64

164. Ong E, Wong MU, Huffman A, He Y (2020) COVID-19 coronavirus vaccine design using reverse vaccinology and machine learning. Front Immunol 11:1-13. https://doi.org/10.3389/ fimmu.2020.01581

165. Magar R, Yadav P, Farimani AB (2020) Potential neutralizing antibodies discovered for novel corona virus using machine learning. Sci Rep. https://doi.org/10.1101/2020.03.14.992156

166. Patankar S (2020) Deep learning-based computational drug discovery to inhibit the RNA Dependent RNA Polymerase: application to SARS-CoV and COVID-19. pp 1-17. https://doi.org/10. 31219/osf.io/6kpbg

167. Alex Z, Bogdan Z, Alexander Z, Vladimir A, Victor T, Quentin V, Dmitry SB, Daniil P, Rim S, Andrey F, Michael B, Steve M, Edgardo L, Deborah B, Keita F, Yen-Chu L, Shih-Hsien H, Hsuan-Jen L, Alex A, Yan I (2020) Potential non-covalent SARS-CoV-2 3C-like protease inhibitors designed using generative deep learning approaches and reviewed by human medicinal chemist in virtual reality. chemRxiv. https://doi.org/10.26434/ chemrxiv.12301457.v1

168. Beck BR, Shin B, Choi Y, Park S, Kang K (2020) Predicting commercially available antiviral drugs that may act on the novel coronavirus (SARS-CoV-2) through a drug-target interaction deep learning model. Comput Struct Biotechnol J 18:784-790. https://doi.org/10.1016/j.csbj.2020.03.025

169. Metsky HC, Freije CA, Kosoko-Thoroddsen T-SF, Sabeti PC, Myhrvold C (2020) CRISPR-based surveillance for COVID19 using genomically-comprehensive machine learning design. bioRxiv. https://doi.org/10.1101/2020.02.26.967026

170. Zhavoronkov A, Aladinskiy VA, Zhebrak A, Zagribelnyy B, Terentiev V, Bezrukov DS, Polykovskiy D, Shayakhmetov R, Filimonov A, Orekhov P, Yan Y, Popova O, Vanhaelen Q, Aliper A, Ivanenkov YA (2020) Potential 2019-nCoV 3C-like protease inhibitors designed using generative deep learning approaches DNA double-strand break repair in mammalian cells. View project Matrix-isolated systems modeling View project. https://doi. org/10.13140/RG.2.2.29899.54569.

\section{Authors and Affiliations}

\section{Jawad Rasheed $^{1}\left[\right.$ - Akhtar Jamil ${ }^{2} \cdot$ Alaa Ali Hameed ${ }^{2} \cdot$ Fadi Al-Turjman $^{3} \cdot$ Ahmad Rasheed $^{4}$}

1 Department of Computer Engineering, Istanbul Aydin University, Istanbul 34295, Turkey

2 Department of Computer Engineering, Istanbul Sabahattin Zaim University, Istanbul 34303, Turkey

3 Artificial Intelligence Engineering Department, Research Center for AI and IoT, Near East University, Nicosia, Mersin 10, Turkey
4 Department of Electrical and Electronics Engineering, Eastern Mediterranean University, Famagusta, Mersin 10, Turkey 